\title{
Generation and dissipation of runaway electrons in ASDEX Upgrade experiments
}

G. Pautasso ${ }^{1}$, M. Dibon ${ }^{1}$, M. Dunne ${ }^{1}$, R. Dux ${ }^{1}$, E. Fable ${ }^{1}$, P. Lang ${ }^{1}$, O. Linder ${ }^{1}$, A. Mlynek $^{1}$, G. Papp ${ }^{1}$, M. Bernert ${ }^{1}$, A. Gude ${ }^{1}$, M. Lehnen ${ }^{2}$, P.J. McCarthy ${ }^{3}$, J. Stober ${ }^{1}$, the ASDEX Upgrade team ${ }^{4}$ and the Eurofusion MST1 team ${ }^{5}$

(1) Max-Planck-Institut für Plasmaphysik, 85748 Garching, Germany; (2) ITER Organization, Route de Vinon sur Verdon, 13115 St Paul Lez Durance, France; (3) Department of Physics, University College Cork, Cork, Ireland; (4) see author list of H. Meyer et al. 2019 Nucl. Fusion 59 112014; (5) see author list of B. Labit et al. 2019

Nucl. Fusion 59086020.

E-mail contact of main author: Gabriella.Pautasso@ipp.mpg.de

Abstract. The paper describes under which plasma and machine conditions runaway electrons (REs) are generated during ASDEX Upgrade plasma disruptions. The REs are created by argon injection to investigate methods of current and energy dissipation. The RE beams are stable and last up to a few hundred milliseconds. The experimental findings are described and made available for the validation of theoretical models. In the following, a simple OD fluid model is used to simulate the observed RE current magnitude and time behavior. In spite of its simplicity, the model is consistent with the measured RE current. The injection of heavy gases into the RE beam is then discussed in some detail: The injected gas penetrates into the low density background plasma and through the beam without ionizing significantly. Therefore its effect on the REs can be tested directly and it is found to be consistent with the known Coulomb collisional theory.

\section{Introduction}

Fusion reactors of the tokamak type must rely on a large plasma current, an elongated plasma and a large major radius for a good confinement and a large fusion energy gain, $\mathrm{Q}$, at affordable costs. ITER has been designed to reach $Q \geq 10$ at a plasma current $I_{p}$ $=15 \mathrm{MA}$ and elongation $k=1.70$ (lower values in table 2 of [1]); the European DEMO1 and DEMO2 tokamaks are being designed to reach $\mathrm{Q}=10$ with $I_{p}=20$ and $22 \mathrm{MA}$, and $k=1.6$ and 1.8 respectively (figure 3 in [2]).

Unfortunately, tokamak plasmas are subject to disruptions caused by MHD instabilities [3]. Moreover, disruptions at reactor-relevant currents are predicted to convert a large fraction of the current into high-energy runaway electrons (REs) because of the large avalanche gain [4]. When quickly lost by the plasma (e.g. because the plasma becomes vertically unstable or/and it suffers of other MHD modes) and deposited on the plasma facing components, the REs can cause significant melting of their surface. Therefore, in order for a tokamak to become attractive as a fusion power plant, disruptions must either be absent or be limited to a few events in the plant lifetime while the avoidance or suppression of post-disruption REs must still be demonstrated.

Methods of avoiding, controlling and suppressing the REs are being investigated experimentally on existing machines. Well diagnosed experiments are indispensable for the 
validation of RE generation and suppression models, which can then be used for the simulation of reactor scenarios. Particularly the quest for RE loss mechanisms during both the thermal quench (TQ) and the RE beam lifetime, and for ways to enhance the losses are being pursued.

In this spirit, RE experiments have been carried out at ASDEX Upgrade (AUG) since 2014 [5]. RE beams do not usually occur in AUG mitigated or unmitigated disruptions and they have to be generated in unusual, otherwise uninteresting, circular and lowdensity plasma scenarios. Argon injection is used to induce a fast current quench (CQ) and the consequent large toroidal electric field. A low density target plasma is chosen to minimize the friction force on the electrons during the CQ, and a circular poloidal cross section is imposed to provide a vertically stable plasma.

The generation of a long-lived RE beam is the prerequisite to study the loss mechanisms of suprathermal electrons, the RE confinement properties, the RE beam MHD stability, its interaction with induced magnetic perturbations and the beam dissipation by injected impurities.

This paper reports on the analysis of experimental measurements during the RE generation and suppression, and on the comparison of these measurements with simplified theoretical models. Its content is organized as follows:

The RE avoidance and mitigation schemes foreseen for ITER, along with the uncertainties relative to the effectiveness of their application, are reviewed in section 2. The AUG RE generation scenario is described in section 3 and the relevant measurements are discussed in section 4. The comparison of the measured RE current generated during the induced CQ with the current calculated with a zero dimensional (0-D) model is outlined in section 5. The measured and calculated effect of high- $\mathrm{Z}$ ( $\mathrm{Z}$ is the atomic number) impurities on the RE current suppression is reported in section 6 .

\section{RE avoidance and mitigation schemes foreseen for ITER}

Since unstable RE beams can deposit their energy onto a small area of the surface of plasma facing components and melt it, they should not form in the first place. In theory, the suppression of the RE generation during the $\mathrm{CQ}$ is achievable by increasing the background electron density above the so-called critical density [3]:

$$
n_{c}\left(10^{20} m^{-3}\right) \simeq 11 E_{\phi}\left(V m^{-1}\right)
$$

where $E_{\phi}$ is the toroidal electric field generated by the $I_{p}$ decay during the CQ.

The avoidance of REs by simultaneously increasing the density and tailoring the $I_{p}$ decay - i.e. by keeping the friction larger than the driving force - was suggested in [6]. It consists of injecting a large amount of deuterium (with a small argon concentration) before the TQ, in order to mitigate the divertor heat load during the TQ and suppress $\mathrm{RE}$ generation. The amount of deuterium necessary for the suppression of the RE seed

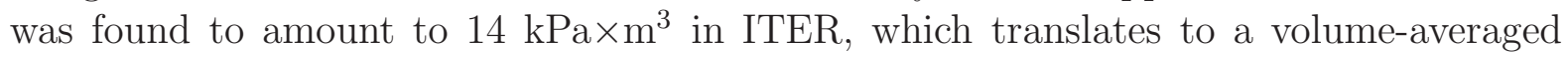
electron density of $4 \times 10^{21} \mathrm{~m}^{-3}$. This density is large but close to the values of the lineintegrated density reached in AUG [7] after massive gas injection (MGI), and in DIII-D 
after MGI and shattered pellet injection (SPI) [8]. In order to suppress REs, the number of electrons must increase before the TQ (within a few ms) over all the flux surfaces, especially in the plasma centre, where the electric field is the largest. Nevertheless, ITER is bigger than existing devices and it is presently not known, whether RE suppression is physically and technically feasible. In addition, this method would not assure RE suppression, if the injected material reached the plasma after the TQ, because of e.g. a late trigger.

The injection of high-Z material is very efficient in dissipating the current of a vertically stable RE beam in middle sized machines (see section 6). In fact, each atom carries many electrons and the RE-ion elastic collisions significantly influence the RE current decay, because of the large cross-section $\left(\propto Z^{2}\right)$. Nevertheless, this scheme might not be suitable for RE suppression in an ITER-like vertically-drifting post-CQ plasma. DINA modelling of mitigated ITER disruptions with RE beam formation and secondary argon injection, have challenged the effectiveness of this RE mitigation scheme [9]. In the simulations, a 15 MA ITER discharge is quenched by adding $10^{19} \mathrm{~m}^{-3}$ argon atoms to the plasma, which develops $\sim 6 \mathrm{MA}$ of RE current. $30 \mathrm{~ms}$ after the TQ, up to $10^{22} \mathrm{~m}^{-3}$ of argon is added to the background plasma, but this second argon injection is found to affect the RE deposition on the wall only weakly for two reasons. Firstly, $30 \mathrm{~ms}$ after the TQ, the plasma has significantly moved vertically (by 2 meters, figure 6 of [9]) and the RE beam is scraped-off on the divertor. Secondly, the second argon injection accelerates not only the current decay but also the vertical drift.

The possibility of controlling the plasma position during the RE beam phase, which requires a smaller elongation and the plasma positioned at the neutral point, would make the RE suppression feasible.

The injection of high-Z impurities into the plasma before TQ, or into the RE beam, for RE suppression, has the advantage that each atom carries many electrons and that the RE-ion elastic collisions significantly influence the RE current decay, because of the large cross-section $\left(\propto Z^{2}\right)$. Nevertheless, high-Z impurities have the disadvantage of causing a too fast $\mathrm{CQ}$ and therefore a large electric field. Whether the massive injection of high-Z impurities, initiated before the TQ and protracted into the RE beam phase, could be a viable solution for RE suppression needs to be studied with experiments and simulations. Moreover, the solution of the RE problem could call for the removal of the constrains now limiting the minimum rate of current decay and the maximum amount of injected gas $[10]$.

It is very uncertain above which plasma current and magnetic energy an ITER disruption will generate significant REs. A major unknown in the problem is the number of energetic electrons, present in the plasma after the TQ, which can exponentially grow in number (avalanche) in the presence of a large electric field (due to the CQ). Several papers ([4] and references within) point out the importance of studying theoretically and experimentally the generation and confinement of energetic electrons, called seed electrons, during the fast quasi-ideal magnetic reconnection phase, i.e. the TQ. A better understanding of this phase would increase the confidence in the simulation of ITER RE scenarios. 


\section{$3 \quad$ Experimental scenario}

\subsection{RE generation and dissipation}

REs have been generated in AUG by injecting between $3 \times 10^{20}$ and $4.8 \times 10^{21}$ atoms of argon into low density circular target plasmas. Larger quantities of argon would induce a short-lived RE beam, uninteresting for the purposes of our studies. $2 \times 10^{20}$ atoms cause a disruption without the formation of a RE beam. Argon was injected with one of the three fast in-vessel valves available on AUG for disruption mitigation studies. Figure 1 shows the position of the valves and of relevant diagnostics in the vessel. The valve in sector 13 is typically used for the first injection, while the valve in sector 4 or the similar valve in sector 6 is used for the second injection. Additional details about the valves can be found in [11].

The injection of neon does not induce any significant RE beam. A possible explanation is that neon cools the plasma less or less rapidly than argon, but simulations are needed to confirm this hypothesis.

The target plasmas had $I_{p}=0.7-0.8 \mathrm{MA}$, a low density (line averaged $\bar{n}_{e}=2-4 \times 10^{19}$ $\mathrm{m}^{-3}$ ), a toroidal magnetic field $B_{t}=2.5 \mathrm{~T}$ and $\sim 2.3 \mathrm{MW}$ of ECRH input power; the plasma equilibrium was kept the same.

Experiments with different values of $B_{t}, I_{p}$ and ECRH power (and therefore electron temperature) are still ongoing and therefore are not discussed here. Discharges with RMP (resonant magnetic perturbation) coils on were discussed in [12]. For sake of completeness, these discharges are shows along with those without RMPs on several scatter plots throughout the paper but, unless specified otherwise, they are excluded from the analysis.

The range of variation of other plasma parameters, relevant for RE generation (see also section 5), are listed in table 1.

\begin{tabular}{|c|c|c|c|c|c|}
\hline$R_{0}(m)$ & $a(\mathrm{~m})$ & $l_{i}(\operatorname{adim})$ & $q_{95}(\operatorname{adim})$ & $k(\operatorname{adim})$ & $T_{e}(0)(\mathrm{keV})$ \\
\hline $1.61-1.63$ & $0.55-0.56$ & $0.95-1.15$ & $3.6-4.1$ & $1.05-1.10$ & $\sim 5$ \\
\hline
\end{tabular}

Table 1. Range of variation of target plasma parameters. $R_{0}$ and a are the major and minor radius, respectively; $l_{i}$ is the internal inductance; $q_{95}$ is the edge safety factor; $k$ is the elongation; $T_{e}(0)$ is the central electron temperature, measured by the Thomson scattering diagnostic.

The injected argon induces a fast $I_{p}$ decay, followed by a long-lived RE beam, carrying a toroidal current of up to $420 \mathrm{kA}$, and lasting up to half a second. Although the target plasmas were similar, different initial RE current values were observed, independently of the amount of argon injected and of other measurable plasma parameters (see section 5). Therefore, a 0-D model is used to discuss the parametric dependencies expected from theory versus those deduced from the experimental measurements.

In some of the RE beams, argon and neon were injected a second time to study their effect on the dissipation of the RE energy and current, and on the MHD stability of the beam. The noble gas is injected into a cold post-disruption plasma with a line integrated density of $\sim 5 \times 10^{19} \mathrm{~m}^{-3}$ and an electron temperature of less than $2 \mathrm{eV}$, 
typically. The plasma density increase after the second injection is discussed in section 4.2.1. Both noble gases accelerate the RE current decay (see figure 2) as expected and the quantitative comparison with theory is presented in section 6 .

\subsection{Plasma position and current control}

The target plasmas were chosen to be circular because they are inherently vertically stable during the discharge and after the TQ. In fact, most of the RE beams remained vertically stable during the slow current decay and the consequent inward radial shift. The position and the shape of the plasma resulting from the magnetic equilibrium reconstructed with CLISTE are in agreement with line integrated Soft X-Ray (SXR) measurements.

The arrows in figure 3 indicate the position of the emission peak, which is consistent with the position of the plasma centre. The plasma control system did not contribute to the vertical stabilization because the control coil currents were part or all of the time saturated. Only in a few cases, a fast vertical displacement did set-in - for reasons not understood - and terminated the RE beam abruptly.

The plasma current is ramped down by the control system. After injection of small amounts of argon, the controlled current follows the pre-programmed time trace (figure 4 (a)). Larger amounts of argon, instead, increase the RE losses, accelerate the current decay and the ohmic heating $(\mathrm{OH})$ coil system cannot keep the actual current at the level of the reference value (not shown).

The RE current does not exhibit a regular monotonic decay but rather a bumpy temporal behavior correlated to sporadic MHD events. A minor MHD event, frequently found to occur after circa $20 \mathrm{~ms}$ from the beginning of the RE beam formation, causes a small current spike and has a $(\mathrm{m}, \mathrm{n})=(1,0)$ non rotating structure (figure 4 (b) and (e)). Diode bolometer and SXR arrays show a redistribution of the central radiation (figure 4 (c) and (f)). The current spike (figure 4 (a) and (d)) suggests a drop of the internal inductance and the flattening of the current profile. The faster decay of the plasma current after the spike is quickly counteracted by the control system with a larger loop voltage.

The final phase of the RE current is often characterized by the faster loss of some tens of kA. Alternatively the current goes to zero with a constant slope. A MHD mode is observed only rarely and in case of the premature termination of the RE beam by vertical instability. Otherwise, the last spike in the Mirnov coils (figure 4 at $1.27 \mathrm{~s}$ ) does not present any poloidal or toroidal asymmetry.

The value of $q_{95}$ from the CLISTE equilibrium reconstruction at the beginning of the RE beam is larger than 10. At the time of the rapid current drop, the edge safety factor $q_{95}$ is not known in general, because the equilibrium reconstruction fails and radiation emission from the beam is not strong enough to allow its own localization. Linear time extrapolation of $q_{\text {edge }}$, toward the end of the RE beam, indicates that its value does not drop below 5 at the time of the final current decay.

In general, the RE beams are quiescent and do not suffer of major MHD instabilities. Therefore they are ideal for the study of RE transport across the magnetic surfaces due to diffusion, turbulence or drifts. 


\section{Experimental measurements}

\subsection{Pre-thermal quench, thermal quench and fast current quench phase duration}

Figure 5 shows the sequence of phases which, initiated at time $t=1 \mathrm{~s}$ by the first argon injection, lead to the RE formation. The durations of these observable phases are briefly discussed here because they influence the RE generation and confinement.

The argon reaches the plasma in front of the valve at $t=1.001 \mathrm{~s}$ (figure $5(\mathrm{~b})$ ), cools the plasma edge and induces the growth of MHD modes (figure 5 (c)), which cause the TQ. We call the phase between the arrival of the first gas at the plasma edge and the TQ onset, the pre-thermal-quench (pre-TQ). The pre-TQ phase lasts some ms and its duration is expected to decrease with increasing number of atoms injected, $N_{i n j}$, as a result of the faster cooling of the plasma edge. This trend is qualitatively found in the experiment and is illustrated by figure 6, which shows the measured pre-TQ durations as function of $N_{i n j} / V_{p}$, where $V_{p}$ is the plasma volume. Additional but still unidentified variables cause the scatter in the figure. The pre-TQ duration is a significant variable for two reasons: firstly, the longer it lasts, the more impurity atoms are injected into and assimilated by the plasma; secondly, the variation of the pre-TQ duration among repeats hints to the somehow unreproducible development of the MHD modes, which then determine the onset of the TQ.

The fast TQ phase lasts typically $0.5 \mathrm{~ms}$, independently of gas and plasma parameters, and can be identified in several diagnostics: the plasma thermal energy is suddenly lost (figure 5 (a)), the central SXR emission drops (figure $5(\mathrm{~d})$ ), the plasma current exhibits a spike (figure $5(\mathrm{a})$ ) and the magnetic fluctuation amplitude grows significantly (figure 5 (c)). The fast decay of the toroidal current carried by the thermal electrons, i.e. the current quench - CQ -, then follows (figure 5 (a)).

Increasing the amount of argon injected, is expected to increase the plasma current decay rate, $\left|d I_{p} / d t\right|$, because larger radiated power induces a lower electron temperature and a larger electrical resistivity. This is indeed observed experimentally and shown in figure 7. The origin of the scatter affecting the data in this figure is not known. Moreover, there is no correlation between $d I_{p} / d t$ and $\Delta t_{\text {pre-TQ }}$, as figure 8 shows. $\left|d I_{p} / d t\right|$ shown in figure 7 was calculated in two different ways, to insure that the choice of the way does not influence the result: (1) as $\Delta I_{p} / \Delta t$ during the first ms (labled "1 ms" in the figure) after the current spike and $(2)$ as $\left(I_{p}\left(t_{T Q}\right)-500 \mathrm{kA}\right) /\left(t\left(I_{p}=500 \mathrm{kA}\right)-t_{T Q}\right)$, where $t_{T Q}$ is the time at the end of the TQ.

The variables $d I_{p} / d t \propto E_{\phi}, T_{e}$ and $Z_{\text {eff }}$ (effective ion charge) influence the RE generation rate and the next question is, whether the magnitude of $I_{R E}$ (which can be measured, while the RE generation rate cannot) is in agreement with theoretical expectations. Section 5 will discuss the answer to this question.

\subsection{Density measurements during disruption}

The RE generation and suppression rates depend strongly on electron, main and impurity ion densities and on the ion charge state (see section 5 and appendix A). Therefore, the density measurements are discussed in detail in this section while the spectroscopy 
measurements were shortly discussed in [5].

Measurements of the free electron density, $n_{e}$, during the disruption and the RE beam lifetime are provided by two diagnostics, namely the $\mathrm{CO}_{2}$ and the DCN interferometers, located in the same toroidal sector, whose geometry is illustrated in figure 9.

The $\mathrm{CO}_{2}$ interferometer relays on a short wavelength and is less susceptible to fringe jumps during fast density transients (e.g. after the first and the second gas injection). In addition, a data analysis technique explained in [13] allows the evaluation of the integrated density also during fast transients with a time resolution up to 200 ns. Two vertical chords are available and view the plasma between plasma centre and edge (figure $9)$.

The DCN interferometer can follow slow transients and provides data every $0.1 \mathrm{~ms}$. It consists of 5 chords (figure 9) viewing the plasma from the LFS midplane. Data analysis based on [14] indicates that the measurements are incorrect during and immediate after MGI but correct again circa 30-40 ms after it.

In the following, both diagnostics will be used to discuss the plasma density evolution in three phases (see figure 10): during and after the first gas (argon) injection, during the $\mathrm{RE}$ beam and during and after the second gas injection.

\subsubsection{Argon assimilation after first gas injection}

The assimilation of argon MGI in AUG (neon assimilation was discussed in [11]) has not been documented in the literature so far and therefore it is discussed here. The argon amounts injected to create the RE beam are relatively small - if compared to the amounts needed to reach the critical density - since larger quantities would suppress its generation. The corresponding fueling efficiency $\Delta n_{e, V-1} /\left(N_{i n j} / V_{p}\right)=60+/-20 \%$ (figure 11) is large and similar to the measured and calculated [15] assimilation of neon in this range of injected number of atoms, $N_{i n j} . \Delta n_{e, V-1}$ is the increase of the electron density, averaged along the $\mathrm{V}-1$ chord of the $\mathrm{CO}_{2}$ interferometer during the $5 \mathrm{~ms}$ following the thermal quench (TQ) (see figure 12). Measurements along the more-peripheral V-2 chord often indicate a density on the high field larger than the core density (figure 11). They suggest an influx of gas from the high field side scrape-off layer or from the inner wall during the TQ and therefore are not used in this analysis. The magnetic equilibrium was available for all the discharges in figure 11, that is the interferometer chord length crossing the plasma and $V_{p}$ are known. The assimilated argon amount, $n_{A r}=\Delta n_{e} / Z_{A r}$, can then be evaluated when the average argon ion charge, $Z_{A r}$, is known (see section 5 ).

Additional uncertainties affecting the densities experienced by the REs are due to the unknown toroidal asymmetry of the density and to attributing the measured density to the confined plasma region.

\subsubsection{Density during the RE plateau}

The electron density of the background plasma is evolving slowly during the RE beam plateau and the DCN measurements are correct again in this phase (30-40 ms after the MGI). Therefore, the different interferometer chords $\left(\mathrm{CO}_{2}\right.$ and $\left.\mathrm{DCN}\right)$ allow the reconstruction of the density profile when the magnetic equilibrium is available. An example is shown in figure 13: the density profile and magnitude do not change significantly over 
$150 \mathrm{~ms}, n_{e} \simeq 1-3 \times 10^{19} \mathrm{~m}^{-3}$ at the separatrix, i.e. rather large, and there is a plasma with a measurable electron density - outside of the last closed flux surface. The density is constrained to become zero at $\rho_{\theta}=2$ by the fitting program.

The scatter plot of figure 14 shows that the average plasma density derived from the $\mathrm{H}-1$ chord, during the RE beam phase of several discharges, is around $6-9 \times 10^{19} \mathrm{~m}^{-3}$ independently of the RE current or of the RE current density (averaged on the poloidal cross section). The line averaged $n_{e}$ is also independent of the amount of the injected argon, which was varied by a factor of 10 (not shown). Therefore, the RE background $n_{e}$ is likely to be the result of particle and energy balance dominated by plasma-wall and plasma-RE interaction, recombination and ionization, recycling and atomic physics processes at very low temperatures, which are not easy to model. The electron temperature is known to be less than $2 \mathrm{eV}$ from spectroscopic measurements [5].

\subsubsection{Argon assimilation after second gas injection}

The injection of noble gas into the RE beam is performed to measure its effect in suppressing the REs. The background plasma density is correctly measured by the $\mathrm{CO}_{2}$ interferometer after the second injection. Figure 15 shows the increase of the line integrated density over circa $10 \mathrm{~ms}$ and the following decrease, due to the plasma shrinking towards the HFS and neutralizing, after the decay of the RE current. The time scale of the density rise is given by the gas delivery rate of the valve. In fact, this time scale is shorter for neon (not shown). The density increase after this second injection is considerably smaller than after the first injection, causing the TQ: figure 16 shows a $\Delta n_{e}$ of the order of $3 \%$ during the first $10 \mathrm{~ms}$ after the valve trigger and a maximum of $10 \%$ increase, before the current vanishes and the whole plasma recombines. The $\mathrm{CO}_{2}$ interferometer chord length $l e n_{V-1}=0.50 \mathrm{~m}$ and $l e n_{V-2}=0.55 \mathrm{~m}$ were used to estimate $\Delta n_{e}$. Striking is the immediate reaction of the $\mathrm{RE}$ current to the gas injection, triggered at $\mathrm{t}=1.07 \mathrm{~s}$ in figure 15, and implies the existence of a very fast transport of the impurity atoms into the plasma or/and an enhanced outwards transport and loss of the REs.

Estimates of the ionization mean free path $(\mathrm{mfp})$ of the neutral gas into the cold background plasma, indicate that the AUG RE background plasma is transparent to the gas species injected, i.e. argon and neon. The ionization $m f p$ is defined as

$$
m f p_{i, I}=v_{I} /\left(\epsilon_{I} n_{e}\right) \quad v_{I}=\sqrt{\gamma T_{I} / m_{I}}
$$

where $v_{I}$ is the velocity of the $I$-type gas, $n_{e}$ is the background electron density, $\epsilon_{I}$ is the generalized collisional radiative coefficient ${ }^{1}$ and $\gamma$ is the adiabatic index $(=5 / 3$ for monatomic gas).

The gas exits the valve with a temperature lower than room temperature, because of adiabatic expansion. Elastic and inelastic collisions with the plasma and neutral particles filling the vessel heat up the gas. Figure 17 shows the magnitude of $m f p$ s of hydrogen and noble gases in the 1-20 eV temperature range and in the $10^{19}-10^{21} \mathrm{~m}^{-3} n_{e}$ range . In particular, it shows

- the ionization $m f p$ of the gases traveling at a speed velocity corresponding to 20 ${ }^{\circ} \mathrm{C}$ through a background thermal plasma with temperature $T$;

\footnotetext{
${ }^{1}$ http://open.adas.ac.uk/
} 
- the ionization $m f p$ of the gases traveling through a background thermal plasma of temperature $T$ and in thermal equilibrium with it;

- the collisional heating $m f p$, defined as $m f p_{h, I}=1 /\left(\pi d_{I}^{2} n_{e}\right)$ where $d_{I}$ is the kinetic diameter of the gas atom. It is smaller than the ionization $m f p$.

For all the gas species, with the exception of krypton, the $m f p$ for $T_{e}<2 \mathrm{eV}$ is larger than $1 \mathrm{~m}$, while the AUG plasma minor diameter is less than $1 \mathrm{~m}$. In addition, the gas atoms heat up while crossing the plasma; their temperature, velocity and ionization $m f p$ increase, and therefore they remain neutral. This means that the increase of the density cannot be explained with a fluid model for gas and plasma and that it must be treated kinetically, which complicates modelling considerably.

Different mechanisms can contribute to the gas ionization and assimilation by the background plasma:

- the low energy part of the neutral velocity distribution;

- the high energy part of the background electron distribution, which is non-Maxwellian; the RE electron themselves do not contribute significantly to the ionization because of their huge velocity and small cross section;

- atomic reactions other than excitation ionization of the gas by the background plasma. Unknown is also, how correct are the $\epsilon_{I}$ coefficients in the $T<2 \mathrm{eV}$ temperature range.

Experiments of RE suppression with high-Z gas injection have been carried out in several middle sized tokamaks (e.g. [16] and [5]) and in JET [17], the largest existing tokamak. In the smaller devices, the RE current is dissipated by the high-Z gas, as expected on the basis of collisional theory (at least qualitatively if not always quantitatively) while in JET the effect is not significant. The difference has not been explained yet. Nevertheless it is known that in DIII-D [16] and AUG [5] the RE beam background plasma has $T_{e}<2$ $\mathrm{eV}$ while the JET plasma has $T_{e}=5-15 \mathrm{eV}^{2}$. Therefore, the $m f p$ of the injected gas is much smaller than the JET plasma dimension, the gas ionizes at the plasma edge and can only reach the RE beam by diffusing towards the plasma centre. The diffusion of ionized gas is much slower than the penetration of neutral particles into the plasma. As exploratory study, the slow density rise after argon-gas injection in a JET-like RE beam has been calculated with the following simple model. The diffusion equation for a ionized gas is solved in cylindrical geometry assuming that a fraction of the gas injected by the valve is ionized at the plasma edge and diffuses into the plasma (see appendix C). The classical diffusion coefficient is used and only the collisions among the ions of the injected gas are considered, while the interaction with the deuterium background plasma is neglected. The evolution of the gas density, penetrating into the plasma, is shown in figure 18: it takes several tens of ms for the gas to penetrate to the plasma centre, where the $\mathrm{RE}$ are confined. In addition, in order to reproduce the density measurements on page 7 and 8 of footnote 2, an assimilation efficiency of the injected atoms $A_{\text {eff }}<1 \%$ must be assumed.

\footnotetext{
${ }^{2}$ C.Reux et al. https://tsdw2018.princeton.edu/2017-talks-presentations/
} 
These considerations are also useful in the case of the use of SPI for ITER. In fact, a SPI system injects a mixture of gas and ice. The RE beam background plasma in ITER will be hotter than 1-2 eV. Therefore, part of the gas could ionize outside of the last closed flux surface and remain around the plasma; the gas entering the plasma will ionize and diffuse towards the core; the frozen fragments sublimate on their way towards the plasma centre and RE beam. At the latest, the fragments will sublimate at the edge of the RE beam and the gas will subsequently diffuse inwards and outwards from that flux surface.

\section{3 $\mathrm{D}_{2}$ pellets in RE beam}

Frozen deuterium pellets have been injected into an AUG RE beam with the purpose of studying the refueling efficiency of the beam background plasma with frozen $\mathrm{D}_{2}$ pellets. AUG is equipped with a centrifuge injecting cryogenic pellets from the HFS [18]. The centrifuge is suitable for the injection of $\mathrm{D}_{2}$ pellets but not of heavier frozen gases. For the experiments reported in [19], neon pellets were injected, and this led to the damage of the so-called stop-cylinder in the centrifuge.

A sequence of $15 \mathrm{D}_{2}$ cryogenic pellets were injected with a frequency of $70 \mathrm{~Hz}$ into a $\mathrm{RE}$ beam with an initial RE current of $0.25 \mathrm{MA}$. The relatively (for AUG plasma refueling purposes) large pellets had a volume of $1.9 \times 1.9 \times 2.0 \mathrm{~mm}^{3}$ containing $5 \times 10^{20} \mathrm{D}_{2}$ molecules each. Figure 19 shows the time evolution of several plasma parameters during the RE beam lifetime. The most striking observations are:

- The RE beam lasts $420 \mathrm{~ms}$, i.e. longer than any other beam created up to now in AUG. It is ramped down by the control system and the RE current follows exactly the prescribed reference current (figure 19 (a)).

- The loop voltage, larger than unity in the first $100 \mathrm{~ms}$ of the beam, drops to and then below zero in the remaining $300 \mathrm{~ms}$. This means that the plasma resistivity has become negligible and the REs do not encounter a significant friction from the background plasma (figure 19 (a)).

- Each pellet clearly induces a spike in the line integrated radiation measured by the diode bolometer chord viewing the plasma core - which indicates that an interaction between pellet and plasma takes place (figure 19 (b)).

- None of the density measurements shows an increase of the electron density after pellet injection. The electron density decreases after the injection of the first pellet and becomes zero after $150 \mathrm{~ms}$ from the beginning of the beam (figure 19 (c)), while the RE current is still $\sim 200 \mathrm{kA}$.

The $\mathrm{D}_{2}$ pellets carry a total amount of $1.5 \times 10^{22}$ deuterium atoms which, if uniformly distributed in the vessel volume of $41 \mathrm{~m}^{3}$ results in a density of $3.7 \times 10^{20} \mathrm{~m}^{-3}$. The question is therefore, why the pellets do not increase the RE background density at all. There is experimental evidence - from diodes and manometers - that the pellets enter the plasma, ablate and that neutral atoms (or molecules) cross the plasma and are detected in the lower divertor. Cryogenic pellets are launched from the HFS along a well defined trajectory. Figures 20 (a) and (b) show, for comparison, the light traces generated by a pellet of the same size ablating in a H-mode plasma and measured by two diode arrays 
(the horizontal and the vertical, respectively, whose geometry is shown in figure 21 (e)) located in the injection sector. At the time of the first $\mathrm{D}_{2}$ pellet injection in the RE beam, the same diode arrays record the measurements shown in figure 20 (c) and (d). In this case the radiation intensity is of one order of magnitude smaller and the radiation is more diffuse. The pellet has the effect of significantly decreasing the plasma radiation in the background plasma after its interaction with it. The background radiation is subtracted in figure 20 and negative values of the radiation emission are visible in the sub-figures (c) and $(\mathrm{d})$.

The radiation traces from the two arrays can be mapped to the plasma equilibrium, and figure 21 (a) shows the locations of the start and of the maximum radiation. According to a simple estimate of energy balance (appendix B), a cryogenic pellet crossing a cold (some eV) background plasma, in the presence of REs, does not ablate but sublimates at the edge of the RE beam. This fact is consistent with the position of start and maximum radiation and with the location of the $\mathrm{RE}$ beam deduced from the radiated power profile measured by the diodes. The bolometers measure radiated power of the order of magnitude of the ohmic power. Moreover, they allow to reconstruct a radiation profile peaked in the plasma centre and shown in figure 21 (b). Since the plasma temperature derived from spectroscopic measurements is of the order of $1.5-2 \mathrm{eV}$, the plasma current is carried mostly by the REs. The only source of heating comes from the collisions of the REs with the background plasma. Therefore it is reasonable to assume that the RE current flows in this same central plasma region, which is significantly radiating, although the large transport coefficient could broaden the radiation profile.

The manometer measurements are consistent with the hypothesis that the pellet and then the neutral gas crosses the plasma with the initial velocity and direction of the pellet. In fact, manometers in the divertor and in the toroidal section of the pellet injection see an increase of the pressure first, before other manometers located in different sectors do. The lack of $n_{e}$ increase after each pellet has been an unexpected observation at the time of the execution of the experiment (May 2017). Nevertheless, a a posteriori literature search reveals that this phenomenon is known: a small amount of hydrogen added to argon glow discharges decreases both argon and electron densities (see [21] and references within). Reference [21] describes a model for the different species present in argon-hydrogen glow discharges with a small $\mathrm{H}_{2}$ percentage. The model attributes the drop of $n_{e}$ to the electron recombination with $\mathrm{ArH}^{+}$and $\mathrm{H}_{3}^{+}$ions, whereas the drop in $\mathrm{Ar}^{+}$is attributed to $\mathrm{H}$-atom transfer of $\mathrm{Ar}^{+}$ions with $\mathrm{H}_{2}$ molecules. This is expected to be true also for an AUG RE background plasma.

Reference [20] also reported that deuterium injection reduces the argon population in the RE beam in DIII-D by a mechanism not fully understood.

\section{RE generation: observations and comparison with theory}

The generation of REs in circular low density AUG plasmas has been reliably attained by means of argon injection. Nevertheless, the amount of RE current generated is not always reproducible although the target plasmas are very similar. Moreover, RE generation is expected to depend on the electron and impurity densities but these dependencies do 
not result from the experimental measurements. Figure 22 (a) and (b) show the value of the initial RE current, $I_{R E, 0}$, (defined as the current at $10 \mathrm{~ms}$ after the arrival of the injected impurities at the plasma edge) versus $N_{i n j} / V_{p}$ and versus the amount of impurity atoms injected during the pre-TQ divided by the plasma volume, $N_{\text {inj }}\left(\Delta t_{\text {pre-TQ }}\right) / V_{p}$, respectively. Although all data points have similar plasma equilibria and dimension, density and heating power (within the ranges reported in table 1), the RE current does not seem to scale with $N_{i n j} / V_{p}$, which varies by a factor of 10 .

An unexpected finding is the evidence that the ECRH power, inadvertently delivered to the plasma after the MGI trigger (i.e. during the pre-TQ and fast CQ) can have an influence on $I_{R E, 0}$ (see figure $22(\mathrm{c})$ ). This influence is difficult to understand because during the pre-TQ the injected gas creates a high density layer around the plasma, which reflects the injected power.

A potential dependence of $I_{R E, 0}$ on the pre-TQ duration, on $d I_{p} / d t$, on $\Delta l_{i}$ and $\Delta I_{p}$ was also checked but not found.

These experimental observations led to the use of the 0-D model outlined in appendix A. $I_{R E}$ is generated by the large electric field associated with the current decay. Therefore the three coupled equations 9 - 11 were explicitly integrated with a small time step to calculate the time evolution of the electric field, the total plasma current and the RE current. Both the Dreicer and the secondary generation mechanisms contribute to the thermal-RE current conversion and are described by known first order differential equations, indicated shortly with $\left[d n_{R E} / d t\right]_{1}$ and $\left[d n_{R E} / d t\right]_{2}$ in appendix A, and outlined in [4]. Loss mechanisms of fast electrons and REs are not activated in the model; this corresponds to the experimental observation that the TQ, or phase of large magnetic perturbation, ends when the fast CQ starts. The integration starts just after the end of the TQ, with a flat plasma current, a cold plasma and no initial RE current. This initial conditions are realistic for an AUG RE generation scenario.

Figure 24 shows the magnitude of the RE density expected by the 0-D model for the following parameter ranges or values: $n_{e}=3 \times 10^{19}-2 \times 10^{20} \mathrm{~m}^{-3}$ and $T_{e}=2-10$ $\mathrm{eV}, I_{p}=0.8 \mathrm{MA}, a=0.5 \mathrm{~m}, R_{0}=1.68 \mathrm{~m}, l_{i}=0.7 ; F_{m a g}=0.8$. The Dreicer and secondary generation mechanisms are strong variables of $n_{e}, T_{e}$ and of $Z_{\text {eff }}=\left(n_{D}+\right.$ $\left.Z_{A r}^{2}\left(T_{e}, n_{e}\right) n_{A r}\right) / n_{e}$. This last quantity was calculated using tabulated values of argon ionization stages, $Z_{A r}$. The background deuterium density was kept fixed at $3 \times 10^{19}$ $\mathrm{m}^{-3}$. For $n_{e}<5 \times 10^{19} \mathrm{~m}^{-3}$ the conversion of the thermal current into $I_{R E}$ is predicted to be $>50 \%$. For $n_{e}>5 \times 10^{19} \mathrm{~m}^{-3}, I_{R E}$ is predicted to depend strongly on $n_{e}$.

The experimental points are superimposed to the $n_{R E}\left(n_{e}, T_{e}\right)$ curves, results of the $0-\mathrm{D}$ model, in figure 24. Two types of symbols can be seen: The blue square data points pertain to a subset of discharges resulting from the intersection of two sets: the set of shots with correct $\mathrm{CO}_{2} n_{e}$ measurements and the set of similar discharges introduced in section 3.1; the yellow circles are the set of similar discharges, with and without available density measurement, for which $n_{e}=0.6 \times N_{i n j} / V_{p}+3 \times 10^{19} \mathrm{~m}^{-3}$. The factor 0.6 is derived from figure 11 .

The measured $I_{R E}$ is expressed as $n_{R E}$ and the measured $n_{e}$ (calculated as in figure 11) is used as a "proxy" for the RE beam electron density background. In fact, the $n_{e}$ relevant to the RE generation cannot be measured in AUG during this very dynamic phase. It is the density in the plasma core, where the RE are created, just after the TQ, during the $1-2 \mathrm{~ms}$ of the fast CQ. It is not known how much of the argon, assimilated outside of 
the $q=2$ surface during the pre-TQ, is convected into the plasma center during the TQ. The experimental data (for which $n_{e}$ measurements exist) are consistent with the 0-D model. The data points (yellow circles) with reconstructed $n_{e}$ span a wide range of $n_{e}$ and lie outside of the model prediction. This suggest that the discharges with the lowest and with the larges amount of injected argon should be repeated to measure the actual $n_{e}$.

In addition, the variability of $\Delta t_{p r e-T Q}$ and of the gas assimilation among similar discharges can also influence the apparent lack of $N_{i n j}$-dependence of $I_{R E, 0}$. Profile and kinetic effects, not taken into account in the 0-D model, should be studied and quantified to confirm this hypothesis.

Assuming an initial population of REs (seed) does not change considerably the result. It was verified that varying the equilibrium parameters within their measured variability ranges does not explain the observed scatter of the $I_{R E, 0}$ values in figure 24 .

The 0-D model can also be used to infer an average $T_{e}$ during the fast CQ. The $d I_{p} / d t$ calculated during the first $\mathrm{ms}$ of the fast $\mathrm{CQ}$ is plotted versus $T_{e}$ in figure 25 (a). The experimental range of $d I_{p} / d t=1.8-2.6 \times 10^{8} \mathrm{~A} / \mathrm{s}$ corresponds, according to calculations, to $T_{e}=3.5-7 \mathrm{eV}$ and $Z_{\text {eff }}=1.7-2.7$, shown in figure 25 (b). Appropriate spectroscopic measurements, not available now, could confirm these values of $T_{e}$ and $Z_{\text {eff }}$.

\section{RE current dissipation by high-Z impurities}

REs lose energy through different known mechanisms: By inelastic collision, mainly with bound electrons, through small angle scattering and transfer of energy to the matter in which they travel; by collision with ions and Bremsstrahlung radiation; by synchrotron radiation. In addition, RE elastic collisions with ion nuclei reduces $I_{R E}$ by deflecting the parallel - to the magnetic field - component of the RE velocity into the perpendicular direction.

In some of the RE beams generated, argon or neon were injected $70 \mathrm{~ms}$ after the first argon puff. The argon quantities were in the range $0.4-2.5 \times 10^{22}$. In addition, $2.1 \times 10^{22}$ and $4.3 \times 10^{22}$ amount of injected electrons atoms of neon were injected, and $0.9 \times 10^{22}$ and $1.18 \times 10^{22}$ of kripton. The RE current decay rates show a clear dependence on the amount of high-Z gas injected, confirming that interaction of REs with high-Z material is an effective $I_{R E}$ dissipation mechanism. Figure 26 shows the speed of RE current decay versus the total amount of electrons injected with the impurity gas (1st and 2nd refer to the first and to the second injection), e.g. $N_{e, i n j}=\left[N_{i n j} \times Z\right]_{1 s t}+\left[N_{i n j} \times Z\right]_{2 n d}$.

The collisional damping rates observed from current decay and inferred from $N_{i n j}$ and density measurements are discussed in this section. The radiative losses, evaluated to be smaller than the kinematic losses, are not discussed in the following. Bremsstrahlung measurements and analysis are reported in [22]; synchrotron measurements were carried out with a diagnostic describes in [23] and are in the process of being analysed.

In [5], only the effect of the high-Z impurities during the RE flattop was discussed. Neither a model for the penetration of the gas into the RE beam and background plasma, nor fluid equations describing the suppression of the RE current by high- $Z$ material were available to the first author at the time of publication. In fact the 0-D model of appendix A cannot be used during the RE flattop because equation 13 is valid only for $E_{\phi}>E_{c}$, 
while during the RE current dissipation $E_{\phi}<E_{c}$.

The critical electric field, $E_{c}$ is a linear function of the plasma density,

$$
E_{c}=e^{3} n_{c} \ln \left(\Lambda_{e, \text { free }}\right) /\left(4 \pi \epsilon^{2} m_{e} c^{2}\right)
$$

Reference [24] suggested

$$
n_{c}=n_{R P} \equiv n_{e, \text { free }}+\frac{\ln \left(\Lambda_{e, \text { bound }}\right)}{\ln \left(\Lambda_{e, \text { free }}\right)} n_{e, \text { bound }} \simeq n_{e, \text { free }}+0.5 \times n_{e, \text { bound }}
$$

which does not take into account the effect of inelastic scattering RE-ion and radiation effects.

Recent kinetic calculations [25] have suggested a larger $n_{c}$, which takes into account the effect of ion nuclei and of radiation. The effective critical field results to be $E_{c, e f f}=$ $n_{\text {eff }} E_{c} / n_{e}$, where

$$
n_{\text {eff }}=n_{e, \text { free }}+\mathcal{K} n_{e, \text { bound }} \quad \mathcal{K}=1-2
$$

The estimation of both $n_{R P}$ and $n_{\text {eff }}$ requires the knowledge of the plasma atomic species and their ionization state.

According to section 4.2.1, the density experienced by the RE during and after the second MGI is calculated as follows: $10 \%$ of $N_{i n j}$ is ionized in the plasma, neon and argon are assumed to be ionized once; the rest $90 \%$ is in the form of neutral gas uniformly distributed in the vessel. Therefore the REs experience a volume-averaged free electron density $n_{e, \text { free }} \sim 0.1 N_{i n j}(t) / V_{p}$ (although the profile of the ionized density is not known and it could peak in the plasma centre) and a volume average neutral gas density $n_{Z 0} \sim$ $0.9 N_{i n j} / V_{v v}$ with $V_{p}=10 \mathrm{~m}^{2}$ and $V_{v v}=41 \mathrm{~m}^{2} . N_{i n j}$ is a function of time, depends on the geometry of the injection valve and it is illustrated in figure 27 (a).

Figure 27 (b) shows the good agreement between the time histories of the measured and of the calculated $I_{R E}$ decay, obtained by integrating in time

$$
\frac{d I_{p}}{d t}=-\left(E_{c}-M_{O H-p} \frac{d I_{O H}}{d t}\right) /\left(L_{p} F_{m a g}\right)
$$

(see Appendix A for the definition of the parameters appearing in the equation). The assumption that $10 \%$ of the injected neon ionizes in the plasma is based on the measured density evolution (figure 27 (c)).

It has been argued in [27], that $E_{\phi}$ approaches $E_{c}$ during the CQ. Our analysis (no figure) shows that there is up to a factor of 2 difference in the magnitude of the two calculated Es. Since the difference is not systematic and there are no other plasma or machine parameters correlated with such a factor, we cannot comment on its origin.

\section{Conclusions}

Experiments of RE generation and suppression, following the onset of plasma disruptions, are conducted in AUG to validate theoretical models, which can then be used to evaluate post-disruption levels of RE current and design RE mitigation schemes in larger devices, like ITER and DEMO. 
REs are generated by argon MGI in low density circular plasmas. The observed $I_{R E}$ is not correlated with the amount of injected gas. Nevertheless density measurements are not available at the minimum and at the maximum of the injected quantities; this result motivates the repetition of these shots. The measured $I_{R E}$ is in agreement with the results of simulations with a $0-\mathrm{D}$ model (which evaluates primary and secondary RE generation) for the discharges with available free electron density measurements.

The large dependence of the RE generation and suppression rate on the density spatial distribution in the plasma along with the lack of detailed knowledge of this distribution will be a clear obstacle for the validation of detailed physics models (e.g fast electron losses due to stochastic magnetic flux surfaces during the CQ).

Deuterium injection, differently from argon, does not increase the electron density of the RE background plasma. Cryogenic $\mathrm{D}_{2}$ pellets enter the plasma, ablate at the edge of the $\mathrm{RE}$ beam and purge the plasma of argon. This phenomenon is known from cold plasma and glow discharge studies. It should be clarified how this behavior depends on the plasma temperatures because ITER (like JET) could develop a RE background plasma hotter than AUG.

High-Z gas injected into the RE beam is fast and efficient in suppressing the RE current in AUG. The cold $\left(T_{e}<2 \mathrm{eV}\right)$ RE background plasma is transparent to the injected gas, which can penetrate into the RE beam on its ballistic time scale. This makes the $\mathrm{RE}$ beam in middle sized machine ideal to test the expected damping rate of RE due to collisions with high-Z gas. JET has a hotter plasma and therefore the gas is expected to ionize close to the plasma edge and to diffuse into the plasma before intersecting the $\mathrm{RE}$ beam. In large tokamaks, both diffusion and assimilation of injected impurity gas is expected to retard and limit the refueling of the plasma centre.

After high-Z gas injection into the RE beam, the inferred electric field is comparable to the calculated critical electric field, as expected from theory, and they agree within a factor of 2 .

Presently, it is unknown whether it will be possible in ITER to increase the electron density fast enough and high enough, before the TQ occurs, to suppress the REs generated during disruptions, and maintain it sufficiently large during the whole CQ, to counterbalance the induced electric field. Experiments on existing tokamaks have pointed out that the process of refueling a post TQ plasma carrying a RE beam depends on the plasma parameters density and temperature and species composition. Simulation of the different gas-plasma-RE interactions must be modelled to draw conclusions on the feasibility of the foreseen RE mitigation schemes.

\section{Acknowledgment.}

The first author wants to thank Prof. Ursel Fantz for a useful discussion on the deuteriumargon chemistry and Linea Hesslow for clarifications on avalanche theory.

This work has been carried out within the framework of the EUROfusion Consortium and has received funding from the Euratom research and training programme 2014-2018 and 2019-2020 under grant agreement No 633053. The views and opinions expressed herein do not necessarily reflect those of the European Commission. 


\section{A Appendix}

The 0-D circuit equation for the plasma current $\left(I_{p}\right)$ is the starting point for the experimenttheory comparison:

$$
E_{\phi}=-L_{p} \frac{d I_{p}}{d t}-M_{O H-p} \frac{d I_{O H}}{d t}-M_{v v-p} \frac{d I_{v v}}{d t}
$$

$E_{\phi}$ is the toroidal electric field acting on the plasma current, $L_{p}$ is the plasma self inductance

$$
L_{p}=\mu_{0} R_{0}\left(\log \left(\frac{8 R_{0}}{a \sqrt{k}}\right)-2+\frac{l_{i}}{2}\right)
$$

( $R_{0}$ and $a$ are the plasma major and minor radius, $k$ is the elongation and $l_{i}$ is the internal inductance), $M_{O H-p}$ and $M_{v v-p}$ are the mutual inductances between the $\mathrm{OH}$ coils (carrying a current $I_{O H}$ ) and the plasma, and between the vacuum vessel structure (carrying a current $I_{v v}$ ) and the plasma, respectively.

The equation is simplified here as

$$
E_{\phi}=-L_{p} \frac{d I_{p}}{d t} F_{m a g}-M_{O H-p} \frac{d I_{O H}}{d t}
$$

with $M_{O H-p} d I_{O H} / d t \sim 4.5 \mathrm{~V} / \mathrm{m}$ in all cases discussed in the following. $F_{\text {mag }}<1$ takes into account that some of the plasma magnetic energy is dissipated in the vessel and in the passive stabilizing loop; it is treated in this work as a parameter.

During the CQ, the RE current is generated by the large self induced electric field $E_{\phi}$. $I_{p}$ is the sum of the current carried by the thermal electron and the current carried by the REs. Only the thermal component of the current is related to $E_{\phi}$ through Ohm's law which, in the 0-D approximation, becomes

$$
E_{\phi}=\rho\left(j_{p}-j_{R E}\right), \quad j_{p}=I_{p} /\left(\pi a^{2} k\right), \quad j_{R E}=c e n_{R E}
$$

where $\rho$ is the electrical resistivity [30], $j_{p}$ and $j_{R E}$ are the total and the RE current densities, $e$ is the electron charge, $c$ is the light speed and $n_{R E}$ is the RE density.

REs are generated by the primary (index 1) and avalanche (index 2) mechanisms described - in the fluid approximation - by the following equations [29]

$$
\begin{gathered}
\frac{d n_{R E}}{d t}=\left.\frac{d n_{R E}}{d t}\right|_{1}+\left.\frac{d n_{R E}}{d t}\right|_{2} \\
\left.\frac{d n_{R E}}{d t}\right|_{1}=n_{e} \nu_{e e}\left(E_{D} / E_{\phi}\right)^{3\left(1+Z_{e f f}\right) / 16} \exp \left(-\frac{E_{D}}{4 E_{\phi}}-\sqrt{\left(1+Z_{e f f}\right) \frac{E_{D}}{E_{\phi}}}\right) \\
\left.\frac{d n_{R E}}{d t}\right|_{2}=n_{R E} \frac{E_{\phi} / E_{c}-1}{\tau \ln \Lambda} \sqrt{\frac{\pi \phi}{3(Z+5)}}\left(1-\frac{E_{c}}{E_{\phi}}+\frac{4 \pi(Z+1)^{2}}{3 \phi(Z+5)\left(\left(E_{\phi} / E_{c}\right)^{2}+4 / \phi^{2}-1\right)}\right)^{-1 / 2}
\end{gathered}
$$

$E_{D}$ and $E_{c}$ are the Dricer and critical electric fields respectively

$$
E_{D}=\frac{n_{e} e^{3} \ln \Lambda}{4 \pi \epsilon_{0}^{2} T_{e}}, \quad E_{c}=\frac{n_{c} e^{3} \ln \Lambda}{4 \pi c^{2} \epsilon_{0}^{2} m_{e}}
$$


and

$\nu_{e e}=e^{4} \ln \Lambda n_{e} /\left(4 \pi \epsilon_{0}^{2} m_{e}^{2} v_{t h, e}^{3}\right)$ is the electron collision frequency,

$v_{t h, e}=\sqrt{2 T_{e} / m_{e}}$ is the electron thermal velocity,

$\tau=\left(c / v_{\text {th,e }}\right)^{3} n_{e} /\left(n_{e, \text { free }}+n_{e, \text { bound }}\right) / \nu_{e e}$, according to [26]

$\phi=1-1.46 \sqrt{r / R}+1.72$ and $r \sim a$,

$\ln \Lambda$ is the coulomb logarithm, $n_{c}$ is the critical density discussed in section 6 ,

$Z_{\text {eff }}=\left(\sum_{I} n_{I} Z_{I}^{2}\right) / n_{e}$ is the effective ion charge, $Z_{I}$ is the charge of the ion of species $I$.

In equation $13, Z$ is used and allows for an effective ion charge acting on the REs which includes elastic collisions.

\section{B Appendix}

A RE can cross a cryogenic pellet of deuterium and simultaneously deposit in the pellet a fraction of its energy. Let's calculate how long a $\mathrm{D}_{2}$ pellet can travel without sublimating in a cold plasma carrying a RE current. It is assumed that the fraction of energy deposited is much smaller than - and therefore does not vary - the RE kinetic energy, which can be verified a posteriori.

The pellet, of dimension $L$, injected with a velocity $v_{p}$, penetrates a length $\mathcal{L}=v_{p} \Delta t$ into the RE beam, until the accumulated energy, deposited by the REs in the pellet, amounts to the sublimation energy.

The survival time $\Delta t$ of a pellet in the presence of a RE current density $j_{R E}$ is given by the energy balance equation

$$
j_{R E} /(c e) L^{2} c \Delta t \mathcal{S} L=n_{D_{2}} \mathcal{E} L^{3}
$$

where

- $c$ and $e$ are the speed of light and the electron charge respectively;

- $\mathcal{S}=\epsilon m_{D_{2}} n_{D_{2}}$ is the linear stopping power, $\epsilon$ is the mass stopping power, which for hydrogen (assumed here to be the same for deuterium) and $1.25 \mathrm{MeV}$ electrons (minimum value of $\epsilon$ ) is $\epsilon=3.8 \mathrm{MeV} \mathrm{cm}{ }^{2} / \mathrm{g}^{3}$;

- $m_{D_{2}}$ is the deuterium molecule mass; $n_{D_{2}}$ is the molecule number density in frozen deuterium;

- $\mathcal{E}=1-1.5 \times 10^{-2} \mathrm{eV} /$ molecule is the sublimation energy of frozen hydrogen [28] (assumed here to be the same for deuterium);

- $v_{p}=560 \mathrm{~m} / \mathrm{s}$ is the pellet velocity,

then $\Delta t=e \mathcal{E} /\left(\epsilon j_{R E} m_{D_{2}}\right)=0.95 / j_{R E}$ s and $\mathcal{L}=530 / j_{R E} \mathrm{~m}$, with $j_{R E}$ in $\mathrm{A} / \mathrm{m}^{2}$.

This means that a pellet sublimates after traveling $53,5.3$ or $0.53 \mathrm{~cm}$ in a RE beam with $j_{R E}=1,10$ or $100 \mathrm{kA} / \mathrm{m}^{2}$ respectively. Since the pellets of figure 19 were injected into a $200 \mathrm{kA}$ RE current beam with a minor radius of circa $20 \mathrm{~cm}$ (average $j_{R E}=1.6$ $\mathrm{MA} / \mathrm{m}^{2}$ ), they are expected to have ablated at the edge of the RE beam. This expectation is consistent with the experimental observations.

\footnotetext{
${ }^{3}$ https://physics.nist.gov/PhysRefData/Star/Text/ESTAR.html
} 


\section{Appendix}

The diffusion equation for ions of species $I$ in cylindrical coordinates

$$
\frac{\partial n_{I}(r, t)}{\partial t}=\frac{1}{r} \frac{\partial}{\partial r} r D_{I}(r, t) \frac{\partial n_{I}(r, t)}{\partial r}+S_{I}(r, t)
$$

was solved explicitly by the finite difference method and the assumptions

$$
\begin{gathered}
D_{I}=\rho_{I}^{2} \nu_{I I}, \quad \rho_{I}=\frac{m_{I} v_{\perp, I}}{e Z_{I} B_{t}}, \quad v_{\perp, I}=\sqrt{2 T_{I} / m_{I}} \quad,[30] \\
\frac{1}{\nu_{I I}}=\tau_{I I}=12 \pi^{3 / 2} \frac{\epsilon_{0}^{2} m_{I}^{1 / 2} T_{I}^{3 / 2}}{n_{I} Z_{I}^{4} e^{4} \ln \Lambda_{I I}} \quad[30] \\
\ln \Lambda_{I I}=23-\ln \left(\frac{Z_{I}^{3}}{T_{I}} \sqrt{\frac{n_{I}}{T_{I}}}\right), \quad T_{I} \text { in eV } \quad 4
\end{gathered}
$$

and a source of particles at the plasma edge of intensity

$$
S_{I}(r, t)=\frac{F(t)}{4 \pi^{2} a R_{0}} A_{e f f} \delta(r-a)
$$

were $F(t)$ is the gas flow from the valve, $A_{\text {eff }}$ is the assimilation efficiency, $a$ and $R_{0}$ are the minor and major radius of the circular plasma.

Figure 28 shows how $D_{I} \propto Z_{I}^{2} n_{I}$ and $\tau_{D_{I}} \equiv a^{2} / D_{I}$ for different plasma parameters. For $n=10^{20} \mathrm{~m}^{-3}$ and $T=10 \mathrm{eV}$, typical of a JET RE background plasma, the $\tau_{D_{I}}$ is still larger than $100 \mathrm{~ms}$, i.e. large for the purpose of dissipating a RE beam by MGI.

\section{References}

[1] M. Shimada et al., Nuclear Fusion, 47 (2007) S1-S7

[2] G. Federici et al., Nuclear Fusion, 57 (2017) 092002

[3] T C Hender et al. Progress in the ITER Physics Basis Chapter 3: MHD stability, operational limits and disruptions 2007 Nucl. Fusion 47 S128-S202

[4] A. Boozer, Nuclear Fusion, 58 (2018) 036006

[5] G. Pautasso et al., Plasma Physics and Control Fusion, 59 (2017) 014046

[6] J.R. Martin-Solis et al., Nuclear Fusion, 57 (2017) 066025

[7] G. Pautasso et al., Plasma Physics and Control Fusion, 52 (2009) 124056

[8] N. Commaux et al., 2011 Nuclear Fusion 51103001

[9] S. Konovalov et al., "Assesment of the runaway electron energy dissipation in ITER", Preprint: 2016 IAEA Fusion Energy Conference, Kyoto, paper EX/P639

\footnotetext{
${ }^{4}$ NRL, Plasma Formulary
} 
[10] M. Lehnen et al., Journal of Nuclear Material, 463 (2015) 39

[11] G. Pautasso et al., Nuclear Fusion, 55 (2015) 033015

[12] M. Gobbin et al., Plasma Phys. Control. Fusion, 60 (2018) 014036

[13] A. Mlynek et al., Fusion Science and Technology, 61 (2012) 2

[14] A. Mlynek et al., Review of Scientific Instruments, 85 11D408 (2014)

[15] G. Pautasso et al, "Disruption mitigation in ASDEX Upgrade with the in vessel valve", in Fusion Energy 2008 (Proc. 22nd Int. Conf. Geneva, 2008) (Vienna: IAEA) CD-ROM file [EX/P9-1] and http://wwwnaweb.iaea.org/napc/physics/FEC/FEC2008/html/index.htm

[16] E.M. Hollmannn et al., Physics of Plasma, 22 (2015) 056108

[17] C. Reux et al., Nuclear Fusion, 55 (2015) 093013

[18] B. Ploeckl and P.T. Lang, Rev. Sci. Instr. 84 (2013) 103509

[19] G. Pautasso et al., Nuclear Fusion, 36 (1996) 1291-7

[20] D. Shiraki et al., Nucl. Fusion 58, 056006 (2018)

[21] A. Bogaerts et al., Physical Review E, Vol. 65, 056402

[22] M. Nocente et al., Review of Scientific Instruments, 89 (2018) $10 \mathrm{I} 124$

[23] B. Espositio, et al., Plasma Phys. and Control. Fusion, 59 (2017) 014044

[24] M. Rosenbluth et al., Nuclear Fusion, 37 (1997) 1355

[25] L. Hesslow et al., Plasma Phys. Control. Fusion 60 (2018) 074010

[26] L. Hesslow et al., Nuclear Fusion 59 (2019) 084004

[27] B.N. Breizman et al., Nuclear Fusion, 54 (2014) 072002

[28] B. Pegourie, Plasma Phys. Control. Fusion, 49 (2007) R87-R160

[29] P. Helander et al., 2002 Plasma Phys. and Control. Fusion 44 B247

[30] J. Wesson, Tokamaks, 1997 Clarendon Press - Oxford 


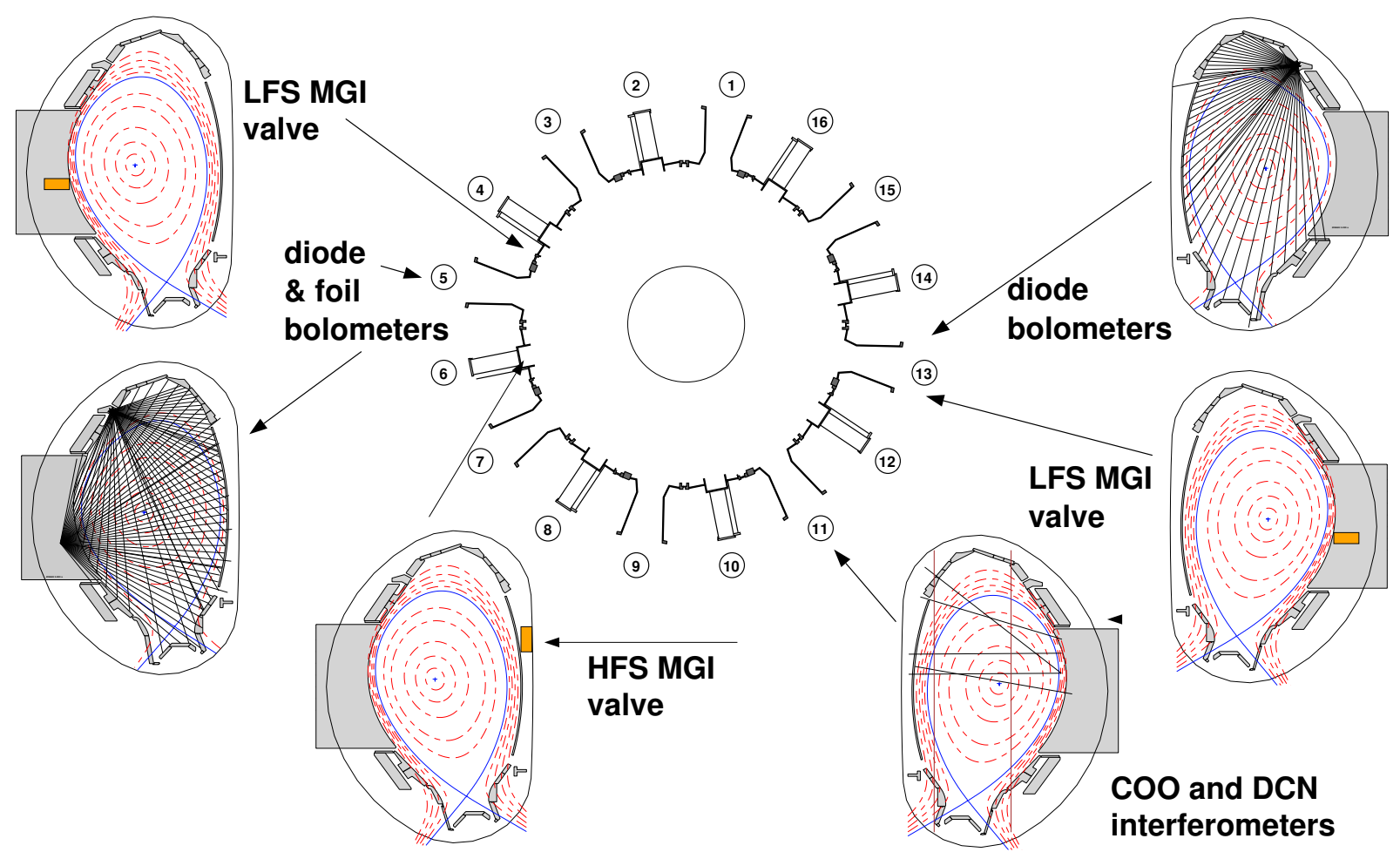

Figure 1: Schematic view of the vessel (from above). A number is associated to each of the 16 toroidal sectors. The location of the MGI valves (orange rectangle) and of relevant diagnostics in the vessel is indicated. 


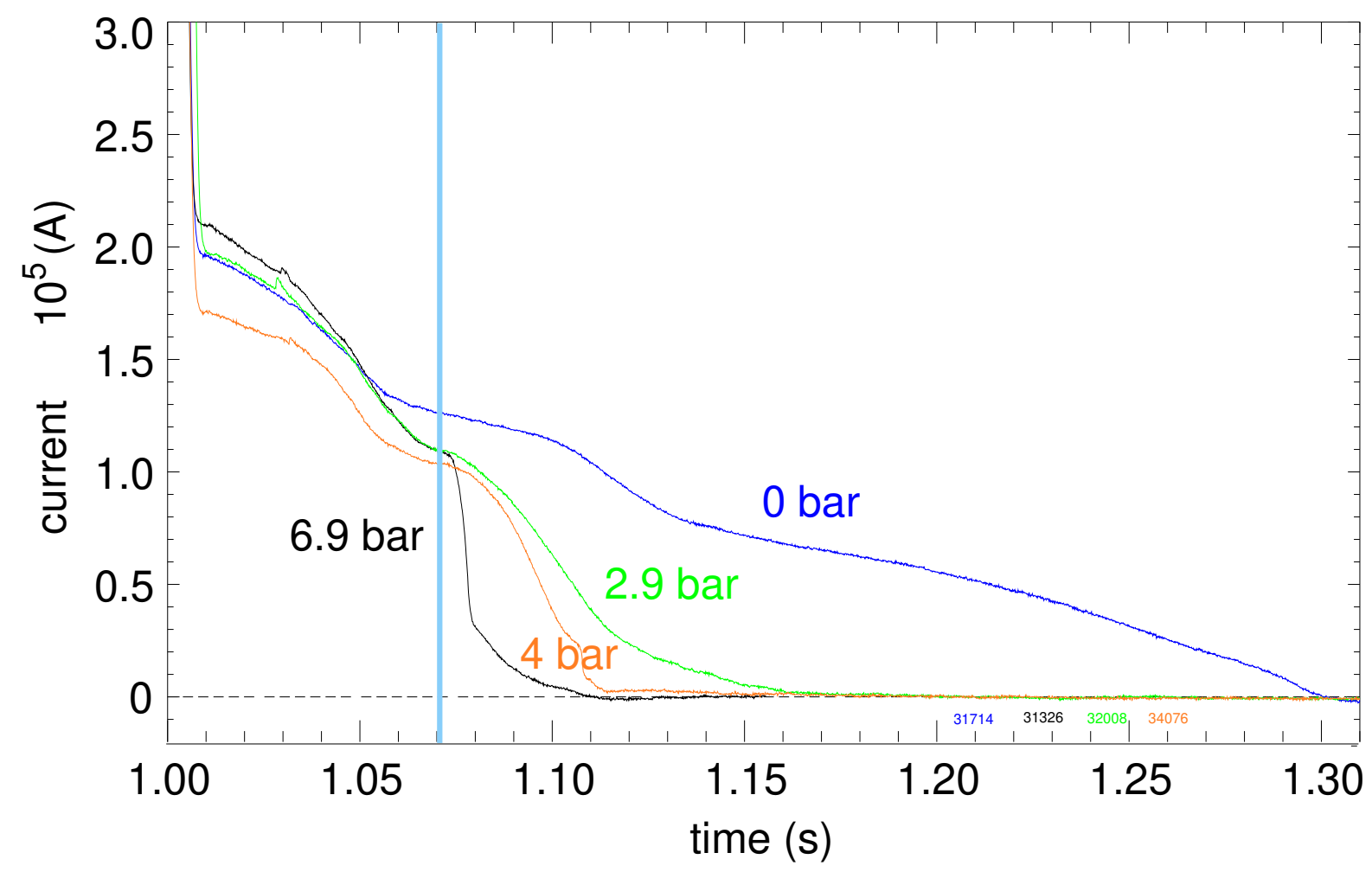

Figure 2: Toroidal current carried by RE beams created with argon injection at $1 \mathrm{~s}$. In three of the beams, argon was injected a second time at $1.07 \mathrm{~s}$. The gas pressure in the valve is indicated in bar. 


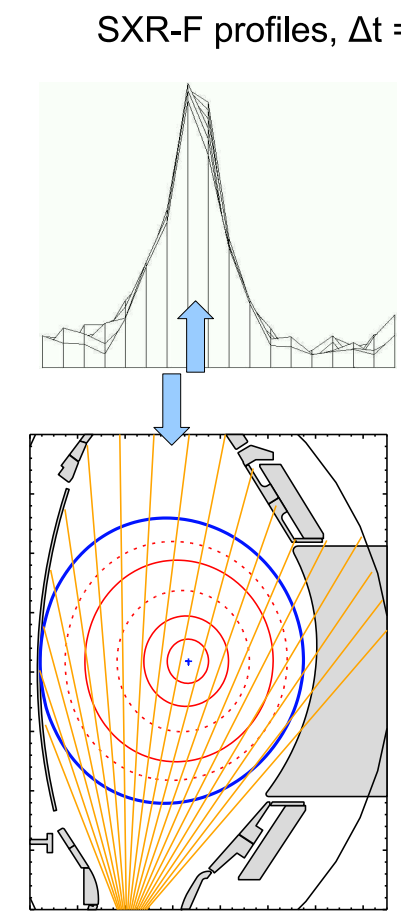

$1.010 \mathrm{~s}$
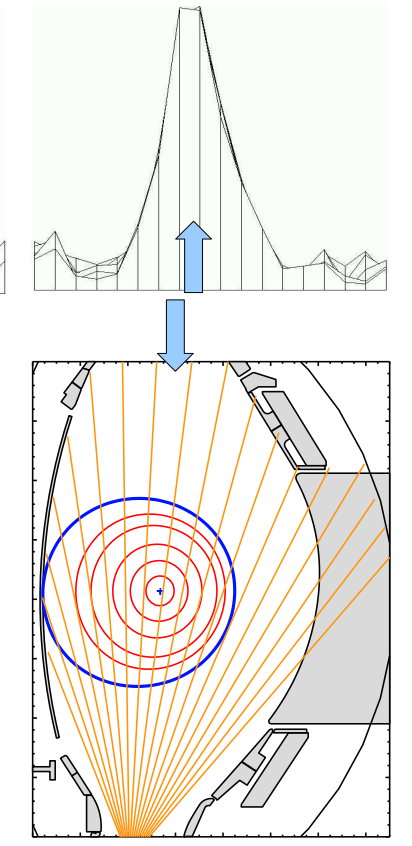

$1.050 \mathrm{~s}$
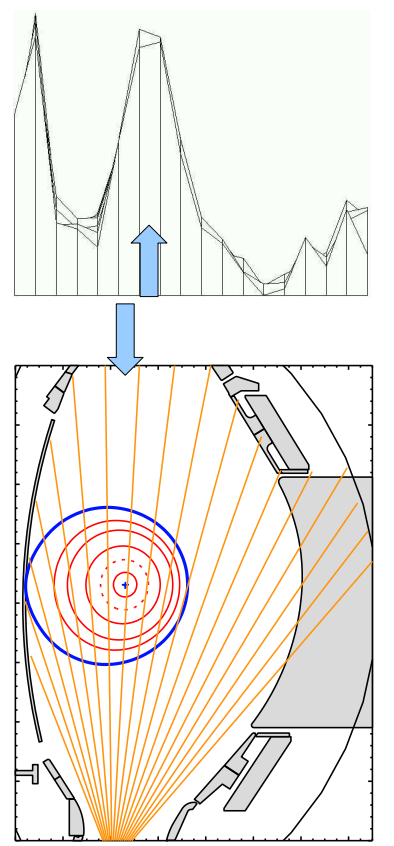

$1.102 \mathrm{~s}$

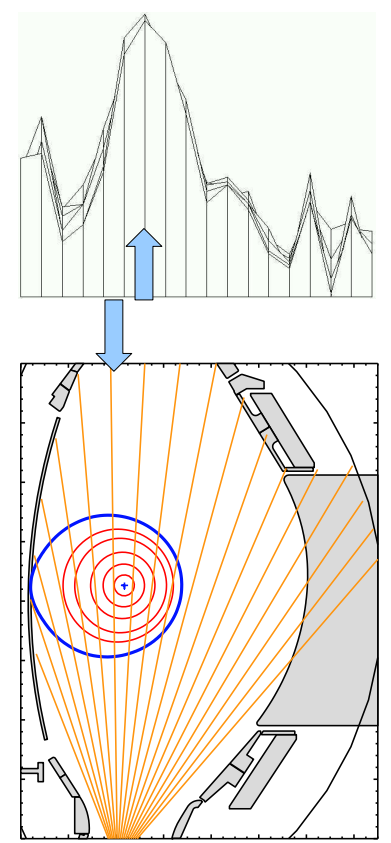

$1.152 \mathrm{~s}$

Figure 3: Evolution of the RE beam magnetic equilibrium and line integrated SXR measurements from a camera, viewing the plasma vertically from the lower divertor. The arrows indicate the position of the emission peak. 

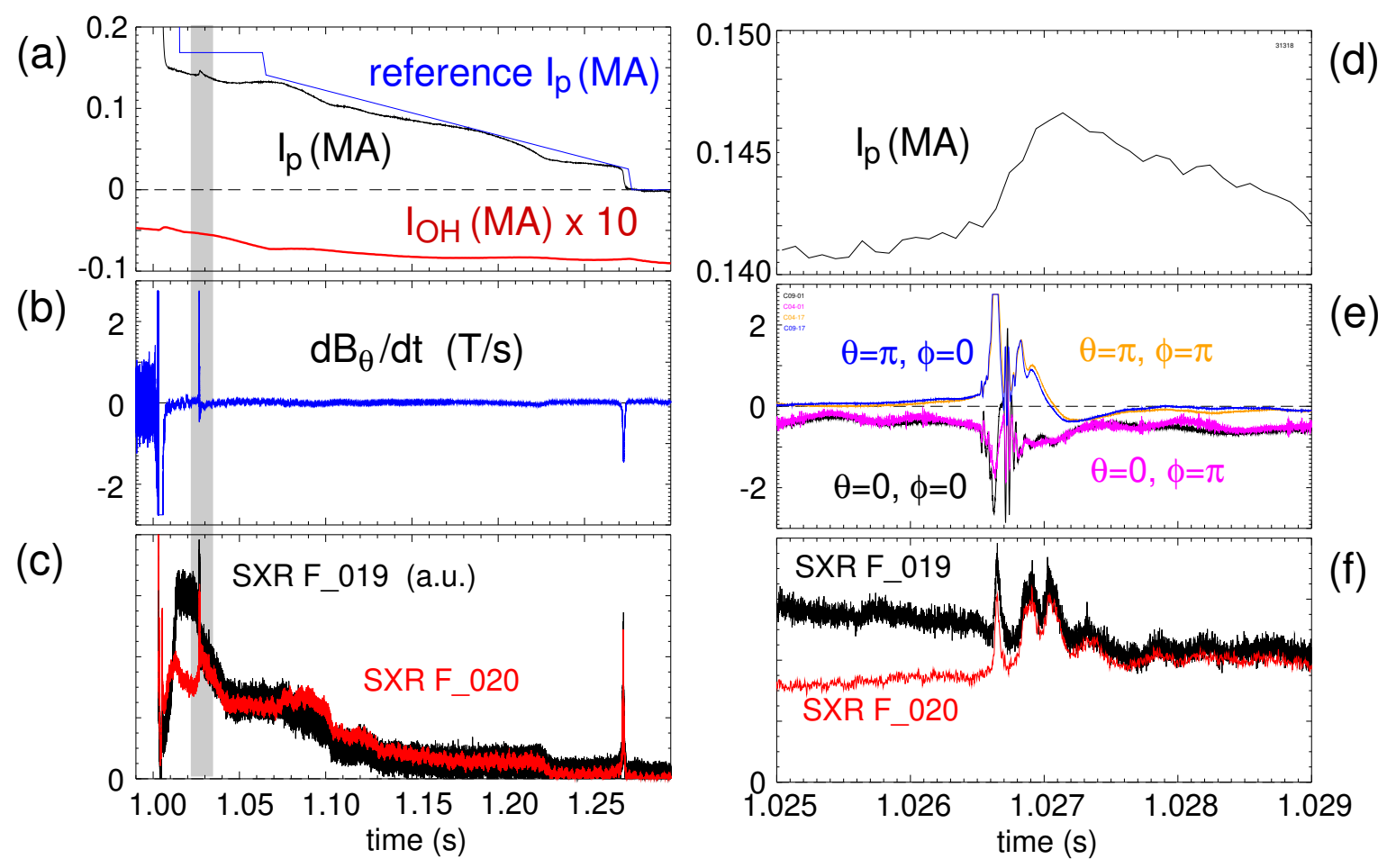

Figure 4: Time traces of (a) pre-programmed and measured plasma current $\left(I_{p}\right)$ and $\mathrm{OH}$ current $\left(I_{\mathrm{OH}}\right)$; (b) one fast poloidal coil; (c) line integrated SXR measurements crossing the plasma core; (d) zoom of (a) in the time interval 1.025-1.029 s showing a spike in $I_{p}$; (e) zoom of (b) showing MHD activity in 4 fast poloidal coils separated poloidally and toroidally of $\pi / 2$; (f) zoom of (c) showing local flattening of the SXR emission (shot 31318). 

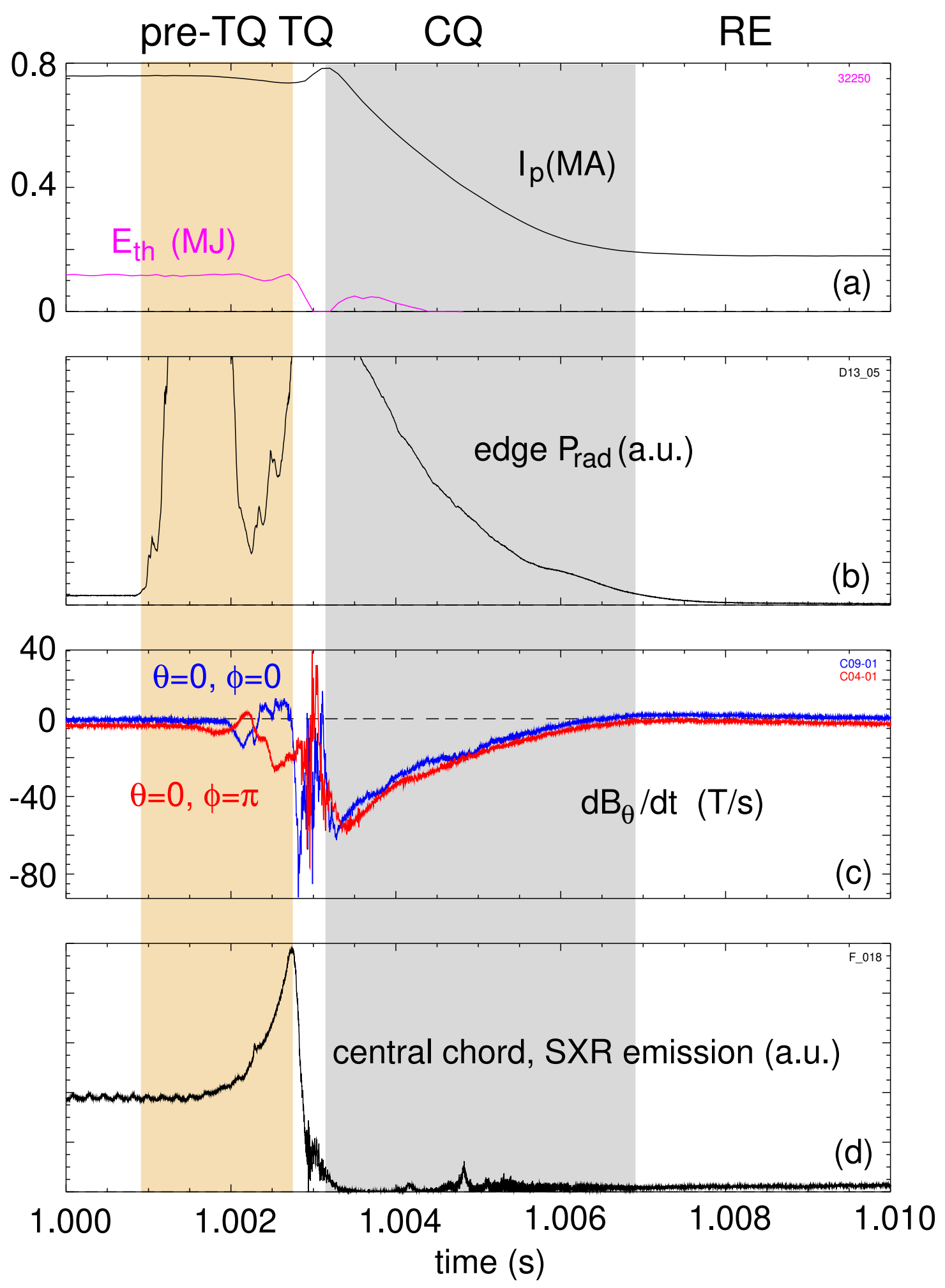

Figure 5: Temporal evolution of several plasma parameters during the pre- thermal quench (pre-TQ), the TQ, the current quench (CQ) and the runaway (RE) phases: (a) plasma current $\left(I_{p}\right)$ and thermal energy $\left(E_{t h}\right)$; (b) line integrated radiated power $\left(P_{\text {rad }}\right)$ from an edge channel of the diode bolometer; (c) fast poloidal coils separated toroidally by $\pi$; (d) line integrated SXR emission from a chord crossing the core (shot 32250). 


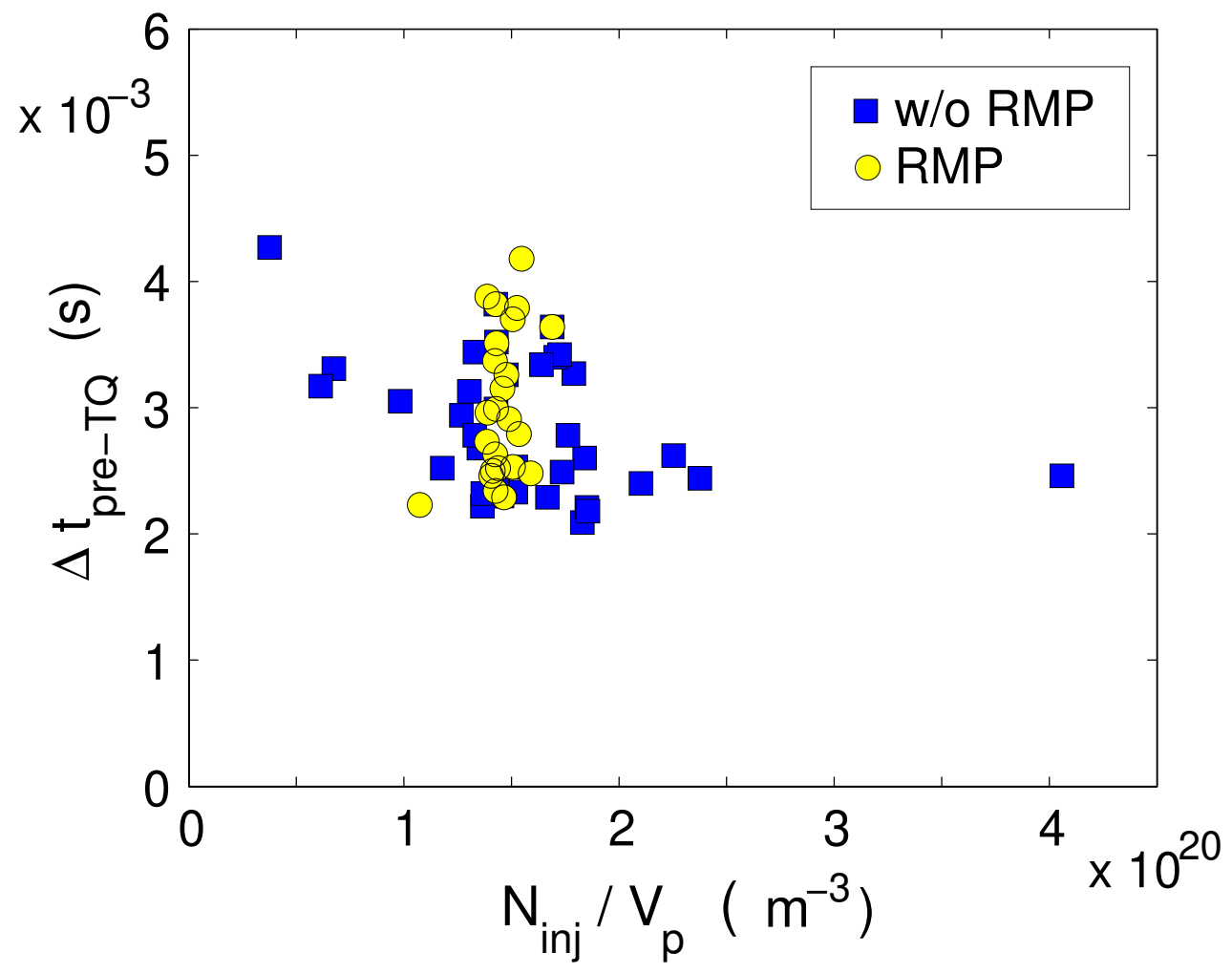

Figure 6: Pre-TQ duration $\left(\Delta t_{\text {pre-TQ }}\right)$ as function of the total amount of injected argon $\left(N_{i n j}\right)$ per plasma volume $\left(V_{p}\right)$ for discharges with and without RMPs. 


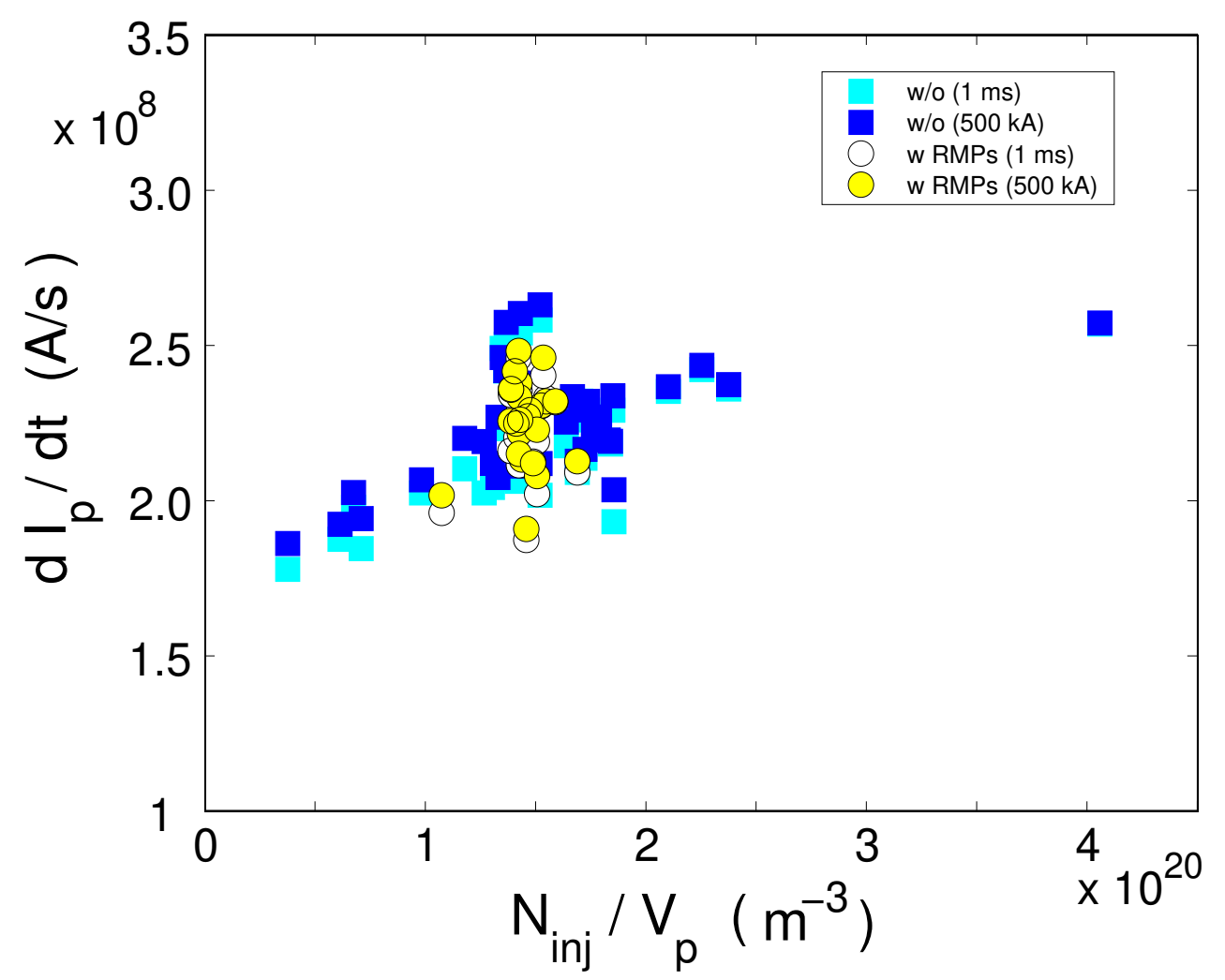

Figure 7: Plasma current decay rate $\left(d I_{p} / d t\right)$ versus the total amount of injected argon $\left(N_{i n j}\right)$ per plasma volume $\left(V_{p}\right)$ for discharges with (w RMPs) and without (w/o) RMPs. $d I_{p} / d t$ is calculated in two different ways (see explanation in text). 


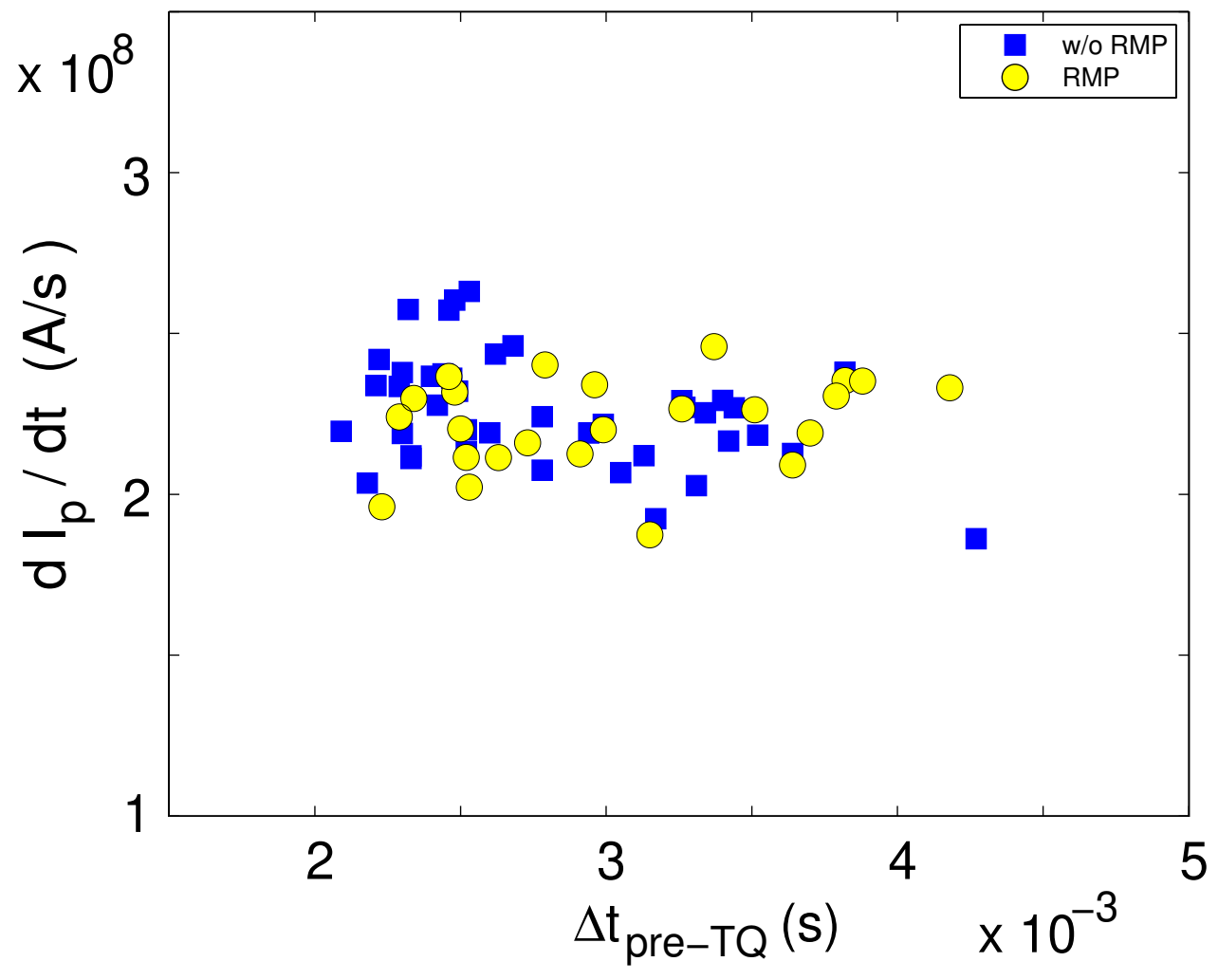

Figure 8: Plasma current decay rate $\left(d I_{p} / d t\right)$ versus pre- thermal quench duration $\left(\Delta t_{\text {pre-TQ }}\right)$. Beams with and without (w/o) RMP on are indicated with different symbols. 


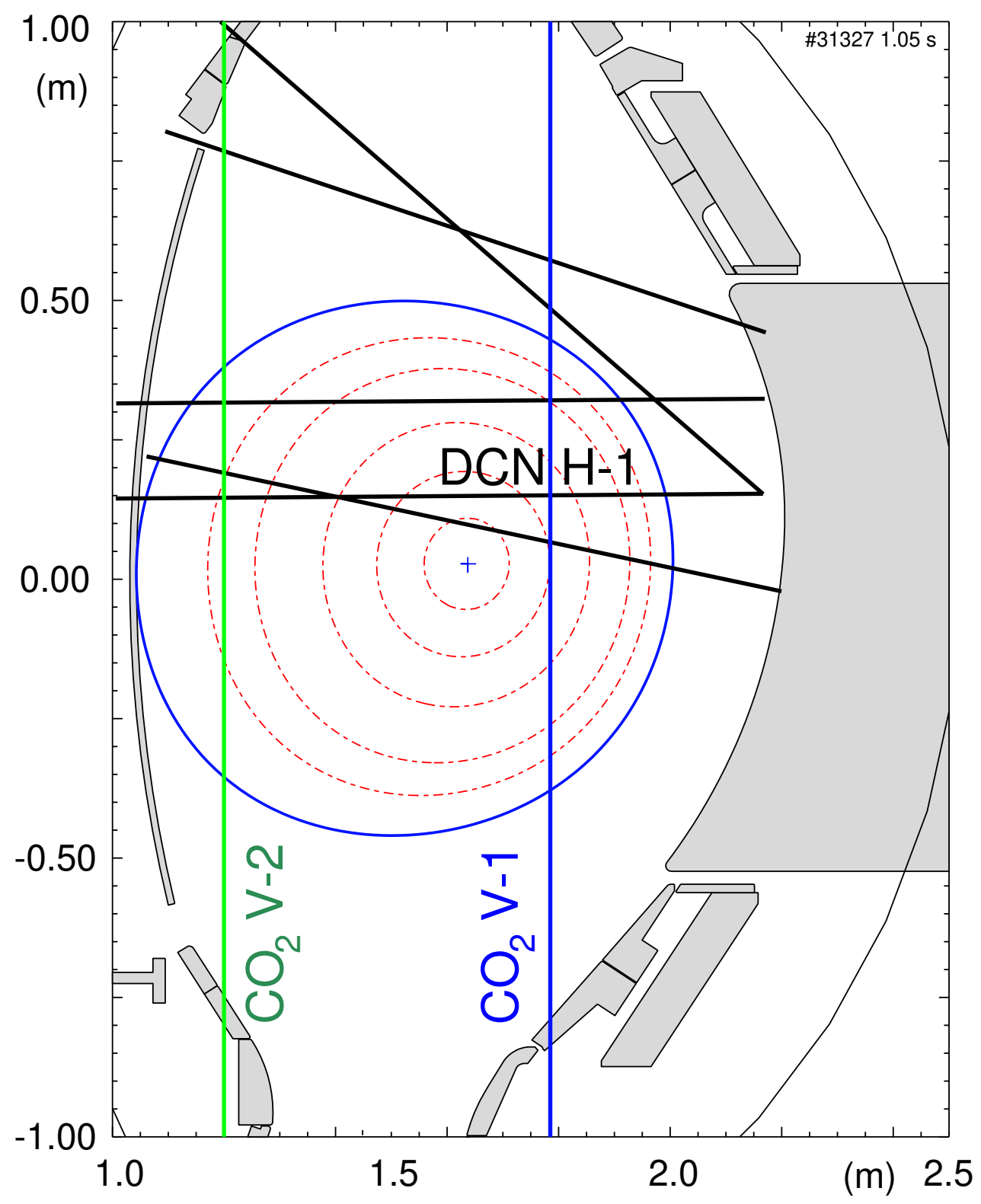

Figure 9: DCN and $\mathrm{CO}_{2}$ interferometer geometry and plasma equilibrium $50 \mathrm{~ms}$ after the TQ (shot 31327). 


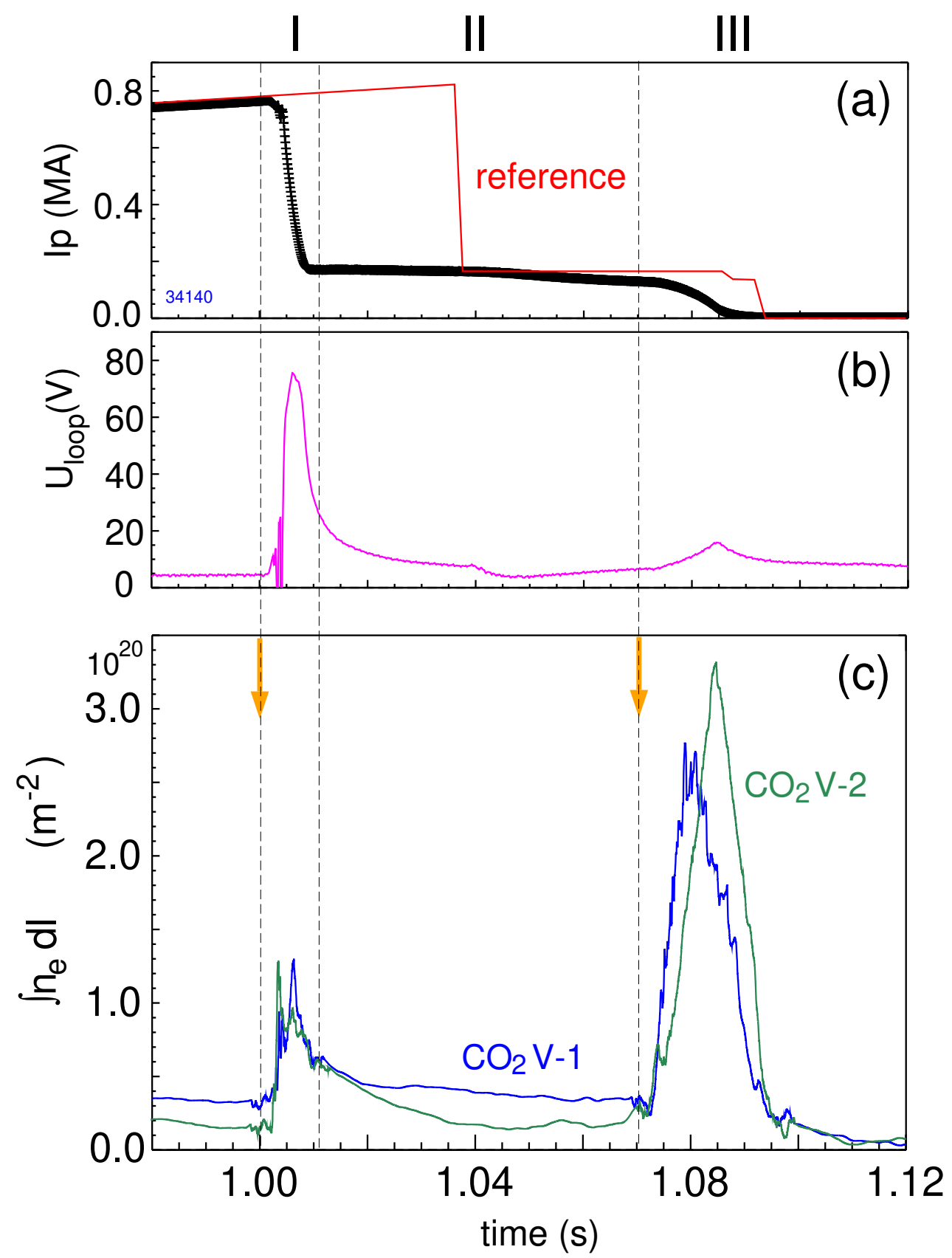

Figure 10: Time traces of (a) the measured and pre-programmed plasma current $\left(\mathrm{I}_{p}\right)$, (b) the loop voltage $\left(\mathrm{U}_{\text {loop }}\right)$, (c) the line integrated electron density along the two $\mathrm{CO}_{2}$ chords. The orange arrows indicate the times at which argon MGI was triggered (shot 34140). 


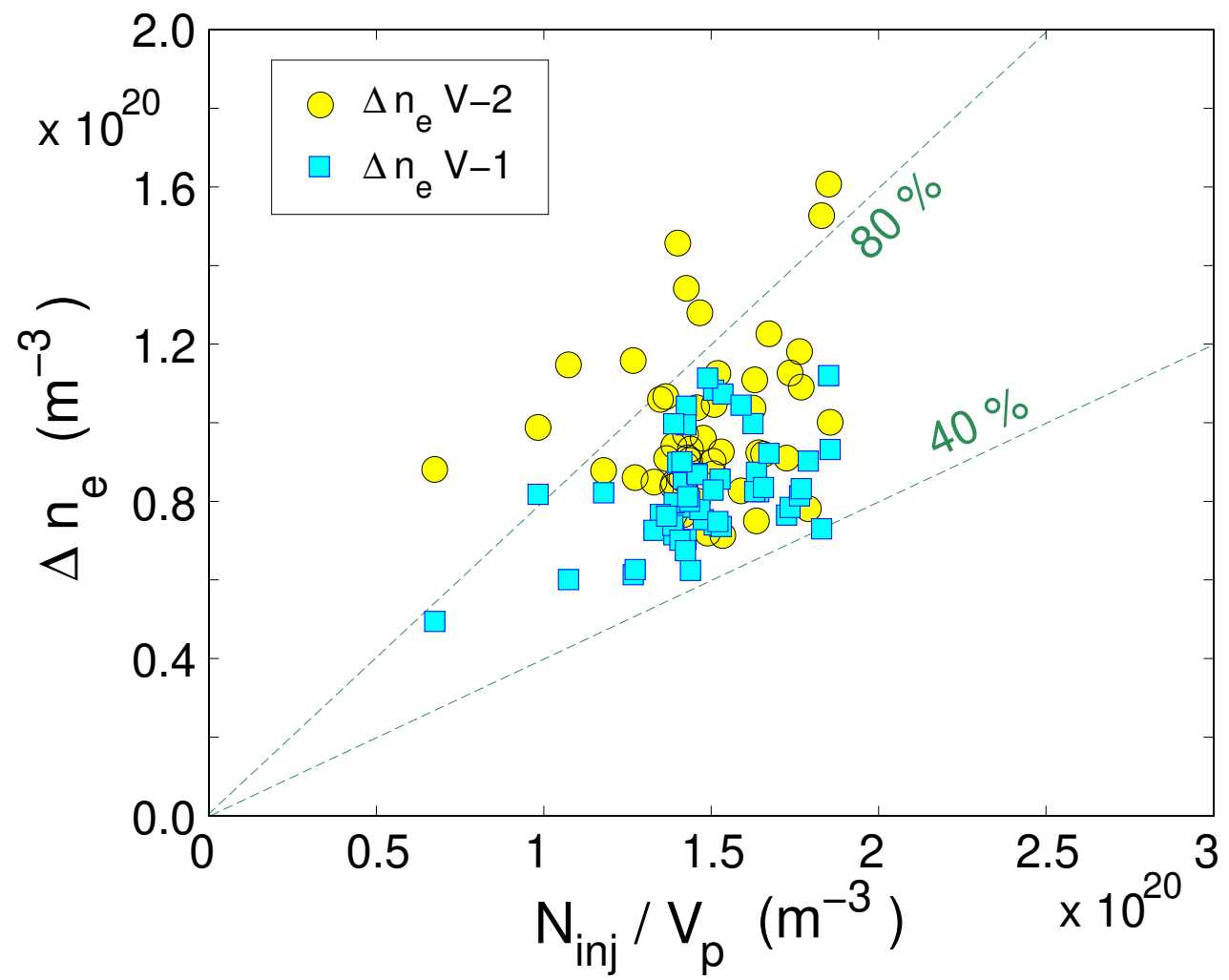

Figure 11: $\Delta n_{e}$ is the increase of the electron density, averaged along the $\mathrm{V}-1$ chord of the $\mathrm{CO}_{2}$ interferometer during the $5 \mathrm{~ms}$ following the thermal quench (figure 12). 


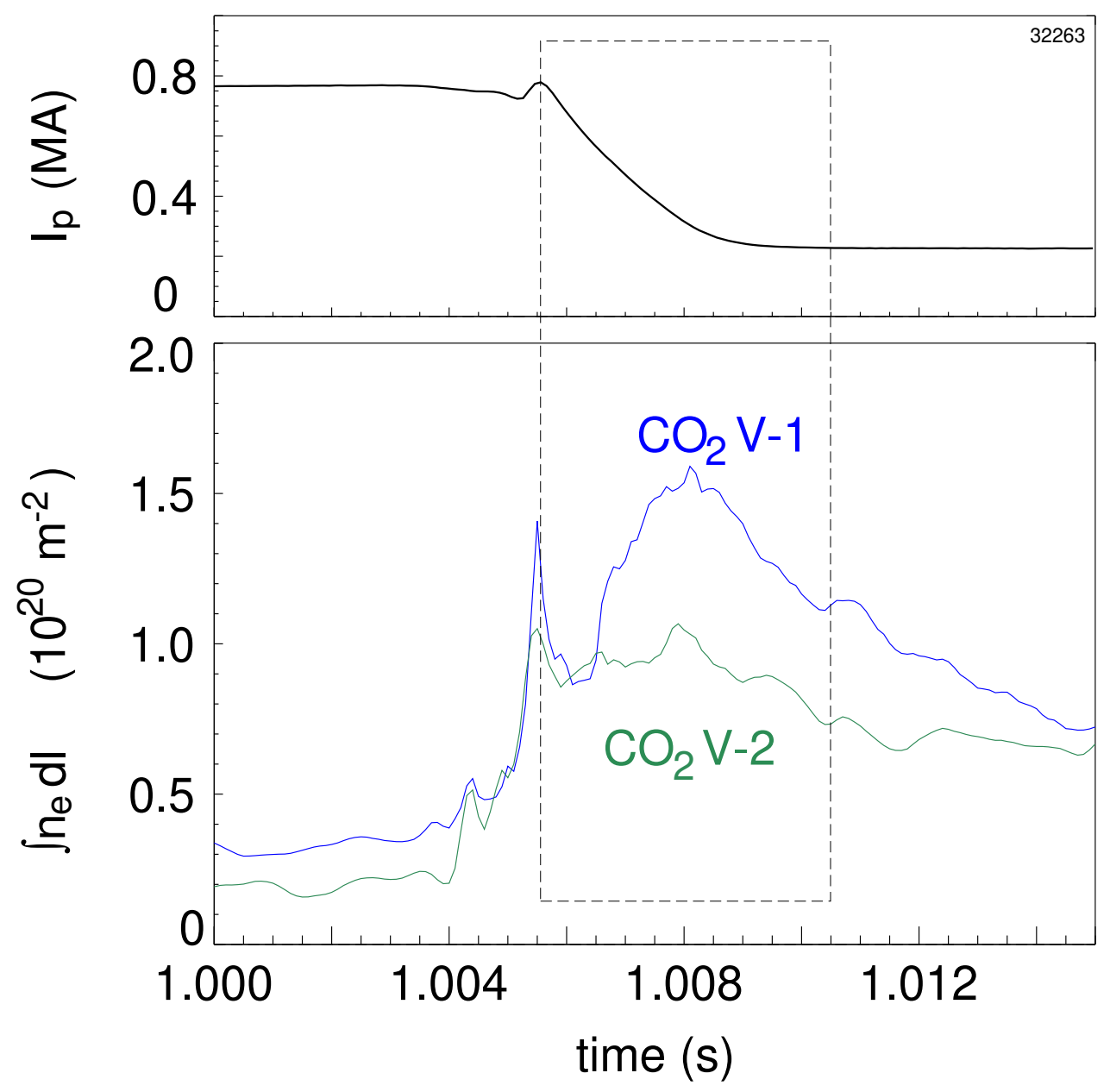

Figure 12: Time traces of plasma current $\left(I_{p}\right)$ and line integrated electron density $\left(\int n_{e} d l\right)$ from the two $\mathrm{CO}_{2}$ interferometer chords, V-1 and V-2. The two vertical lines delimit the time interval of $5 \mathrm{~ms}$ after the thermal quench (shot 32263). 


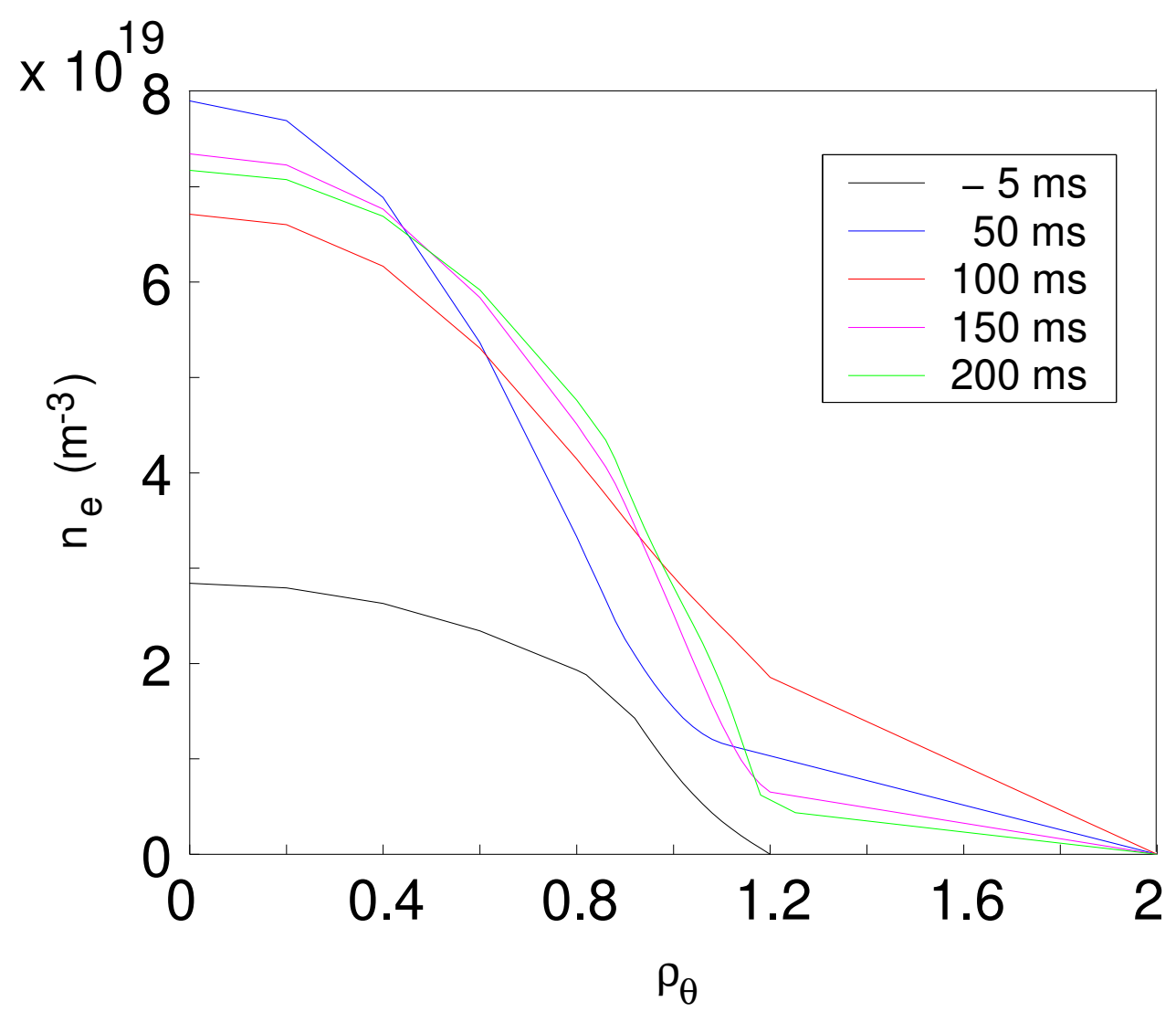

Figure 13: Density profiles $\left(n_{e}\right.$ versus the dimensionless effective radius $\rho_{\theta}$ ) reconstructed using the $\mathrm{CO}_{2}$ and DCN interferometer at different times after the thermal quench (shot 31716). 


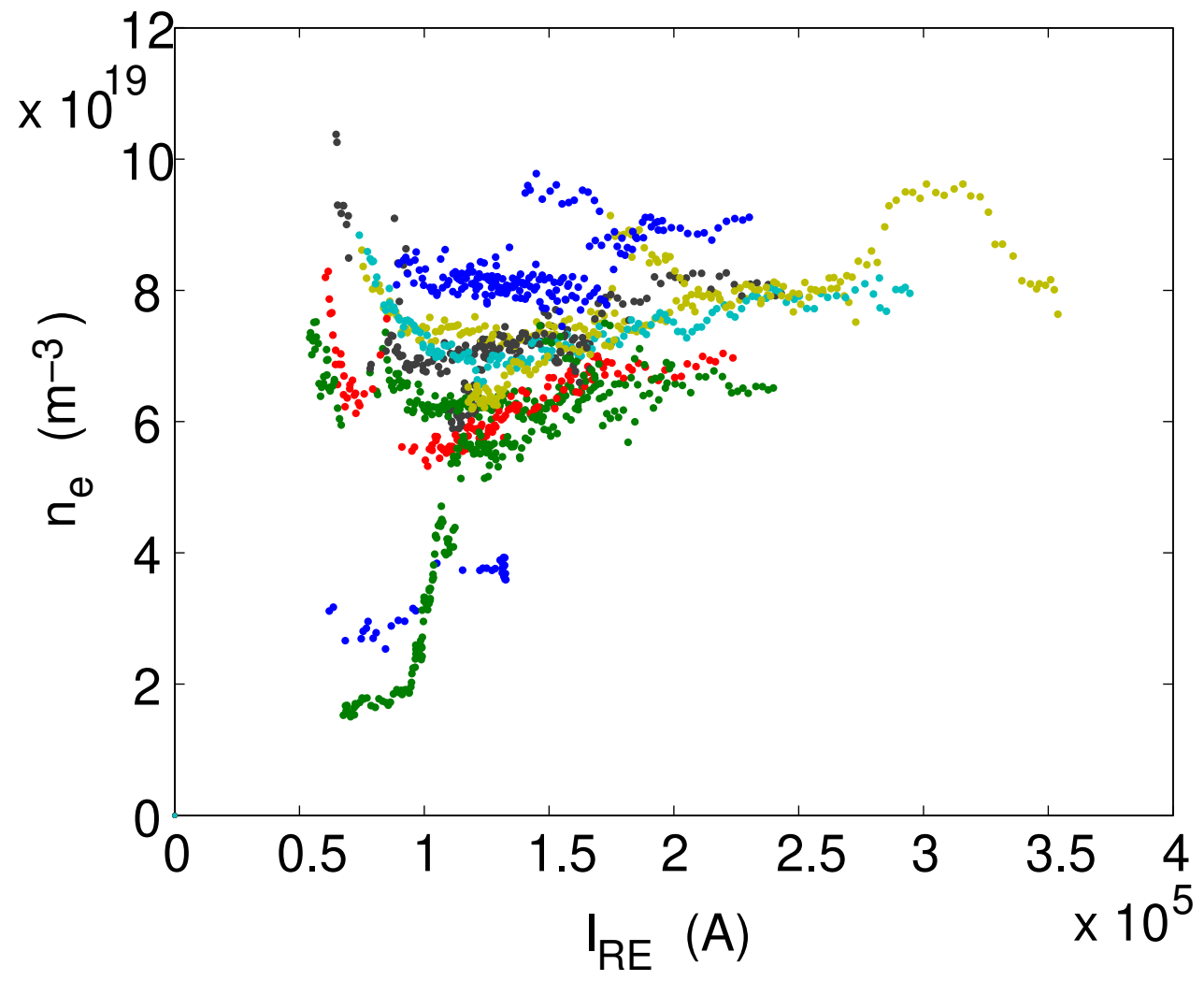

Figure 14: Line averaged electron density $\left(n_{e}\right)$ as measured by the DCN H-1 (figure 9) chord versus RE current $\left(I_{R E}\right)$. Different colors have been assigned to different plasma discharges. 

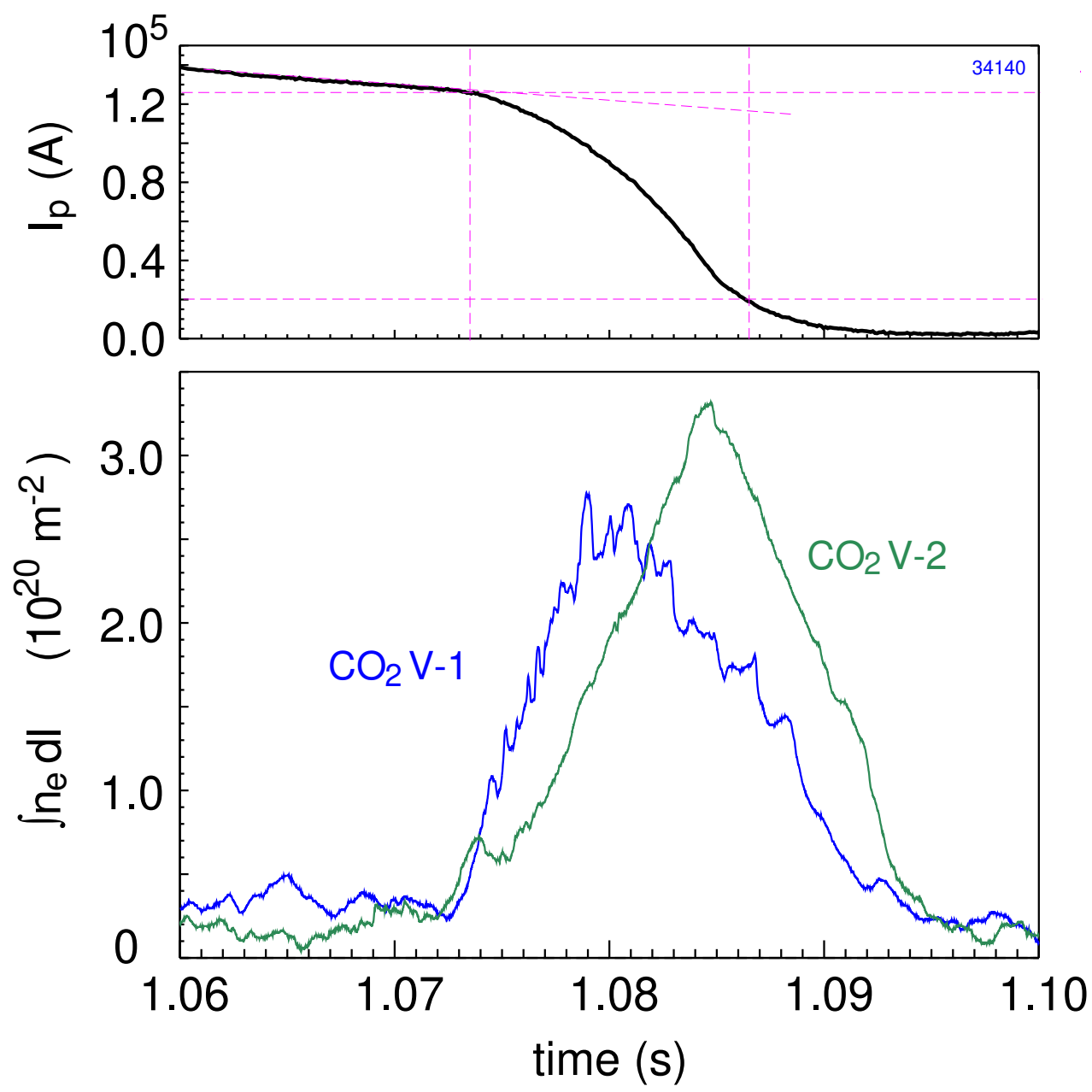

Figure 15: Time traces of plasma current $\left(I_{p}\right)$ and line integrated electron density $\left(\int n_{e} d l\right)$ from the two $\mathrm{CO}_{2}$ interferometer chords, $\mathrm{V}-1$ and $\mathrm{V}-2$. Argon injection is triggered at $\mathrm{t}$ $=1.07 \mathrm{~s}($ shot 34140$)$. 

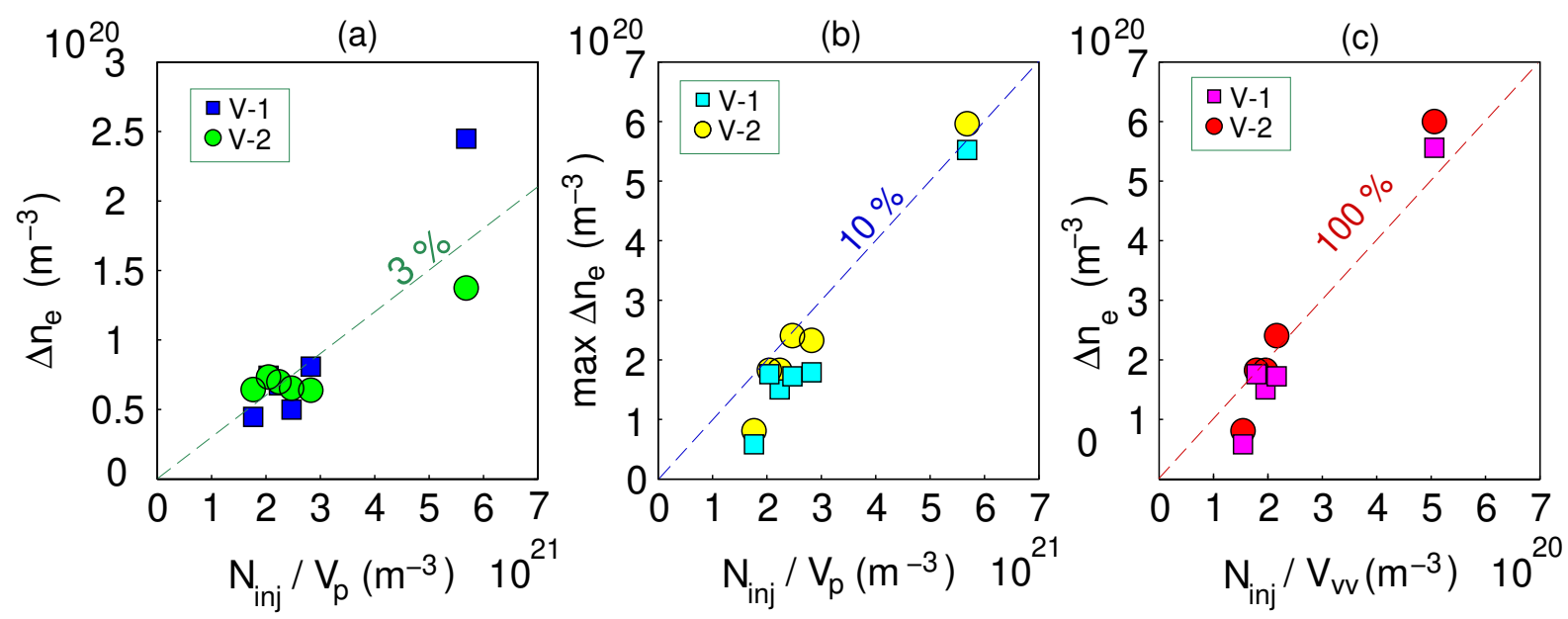

Figure 16: Electron density increase $\left(\Delta n_{e}\right)$ after gas injection into the RE beam (a) during the $10 \mathrm{~ms}$ after 2 nd trigger and versus $N_{i n j} / V_{p}$, where $V_{p}=3.5 \mathrm{~m}^{3}$ is the plasma volume. (b) Maximum value of $\Delta n_{e}$ versus $N_{i n j} / V_{p}$. (c) Maximum value of $\Delta n_{e}$ versus $N_{i n j} / V_{v v}$, where $V_{v v}$ is the vessel volume. 

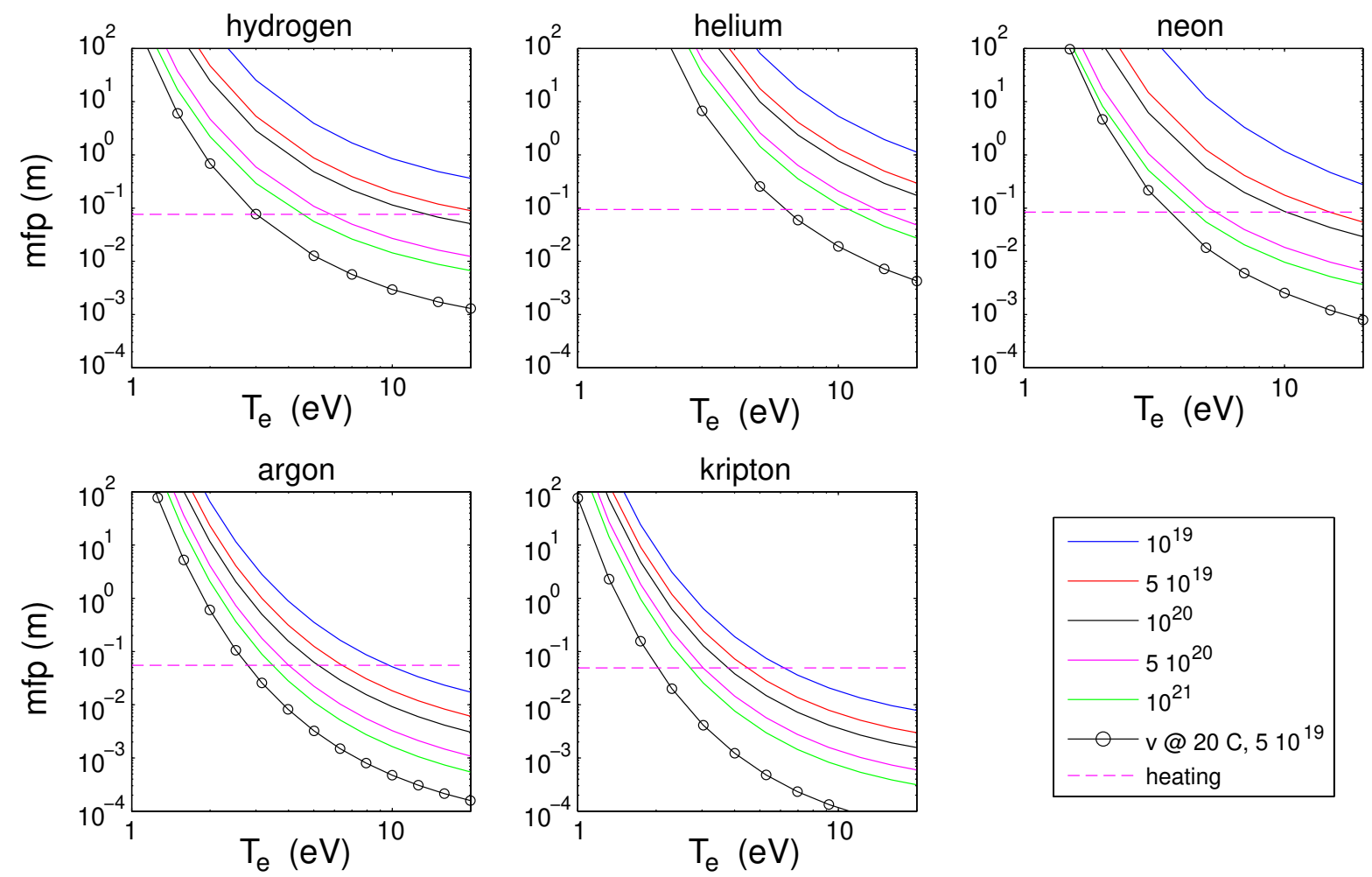

Figure 17: Values of ionization and heating mean free path $(m f p)$ versus electron temperature $\left(T_{e}\right)$ for deuterium and selected noble gases. 

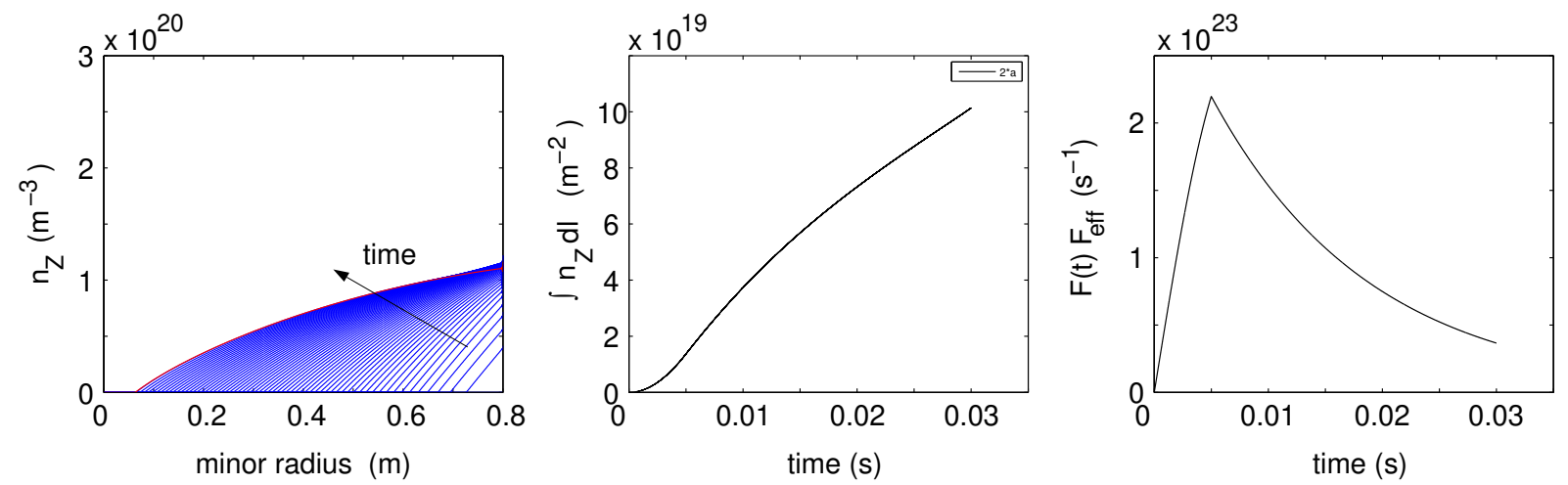

Figure 18: Left: Time evolution $(0-30 \mathrm{~ms})$ of the impurity density profile following the diffusion of argon in a JET like plasma. Centre: Total number of assimilated argon atoms. Right: Particle flow from the valve. 


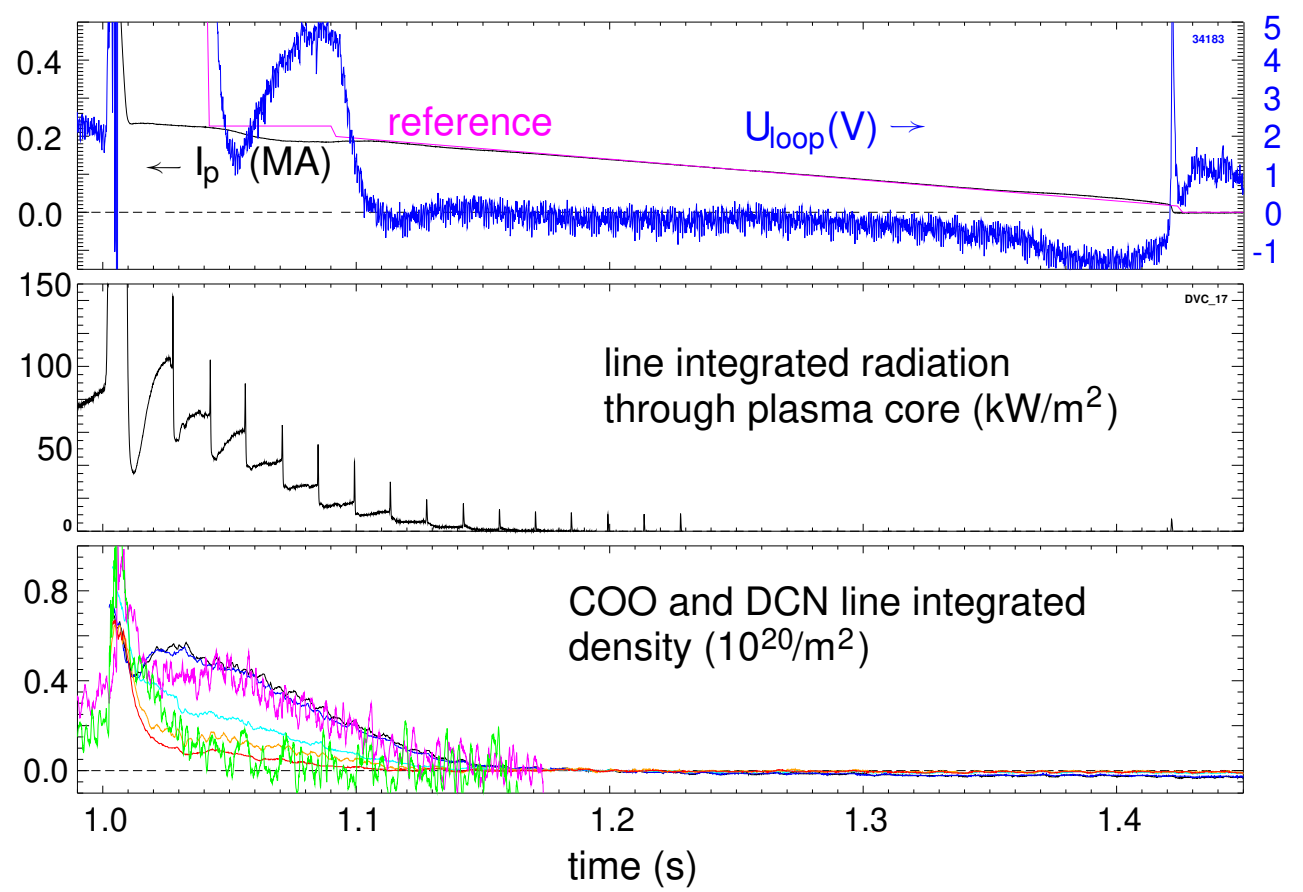

Figure 19: Time traces of plasma parameters during $D_{2}$ pellet injection into a RE beam. Top: Pre-programmed and measured plasma current $\left(I_{p}\right)$, and measured loop voltage $\left(U_{\text {loop }}\right)$. Centre: line integrated radiadion from a fast diode bolometer chord crossing the plasma core. Bottom: several measurements of line integrated density from the $\mathrm{CO}_{2}$ and DCN interferometer (shot 34183). 
(a)

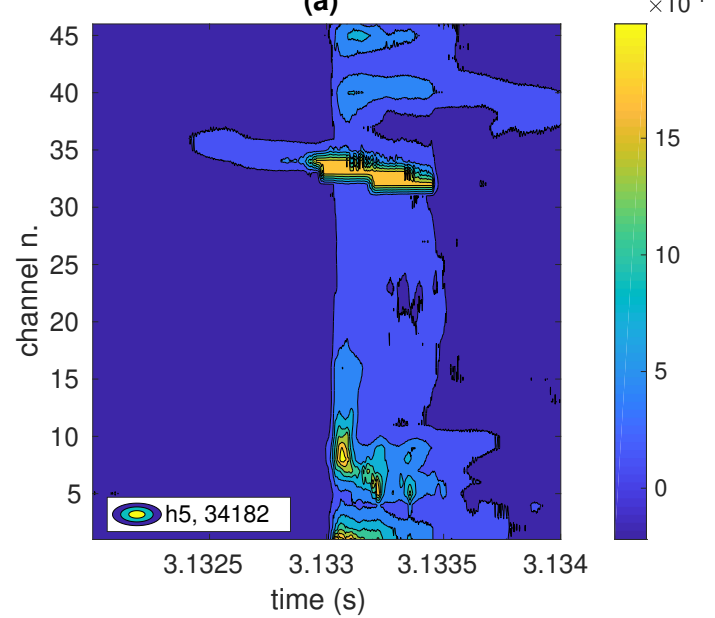

(c)

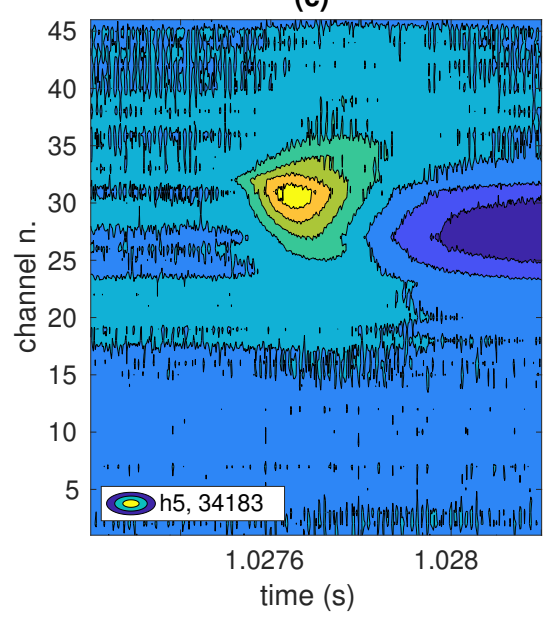

(b)

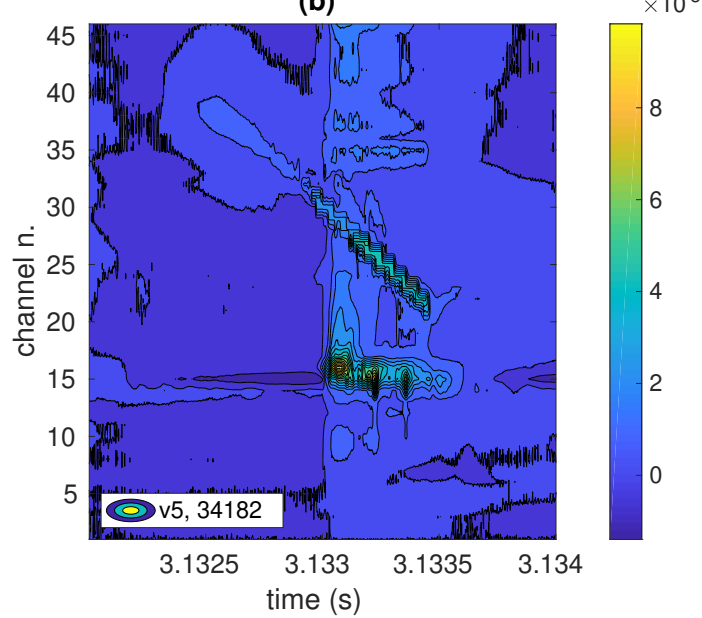

(d)

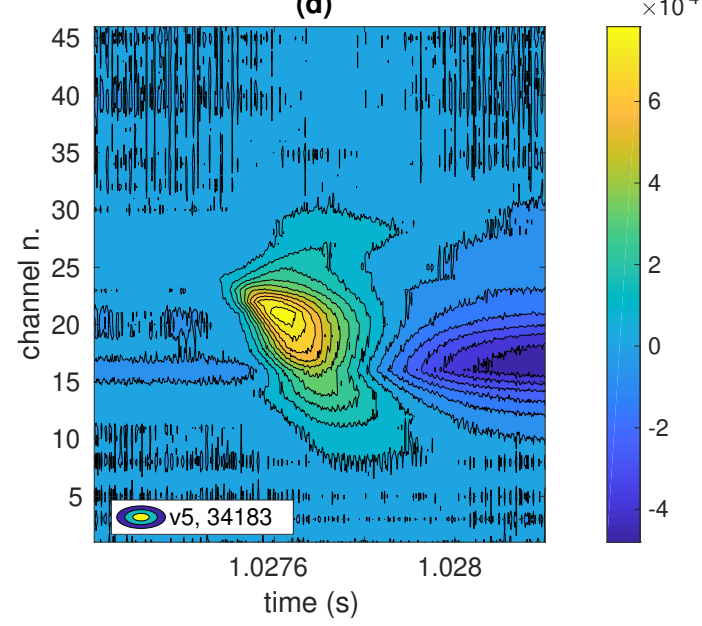

Figure 20: Line integrated measurements from the horizontal (h5, (a) and (c)) and from the vertical (v5, (b) and (d)) diode bolometer cameras. The images show the radiation emission during the ablation of a $\mathrm{D}_{2}$ pellet launched into an $\mathrm{H}$ - mode plasma (top, shot 34182)) and into the RE beam (bottom, shot 34183). Figure 21 (bottom) shows the camera geometry. 

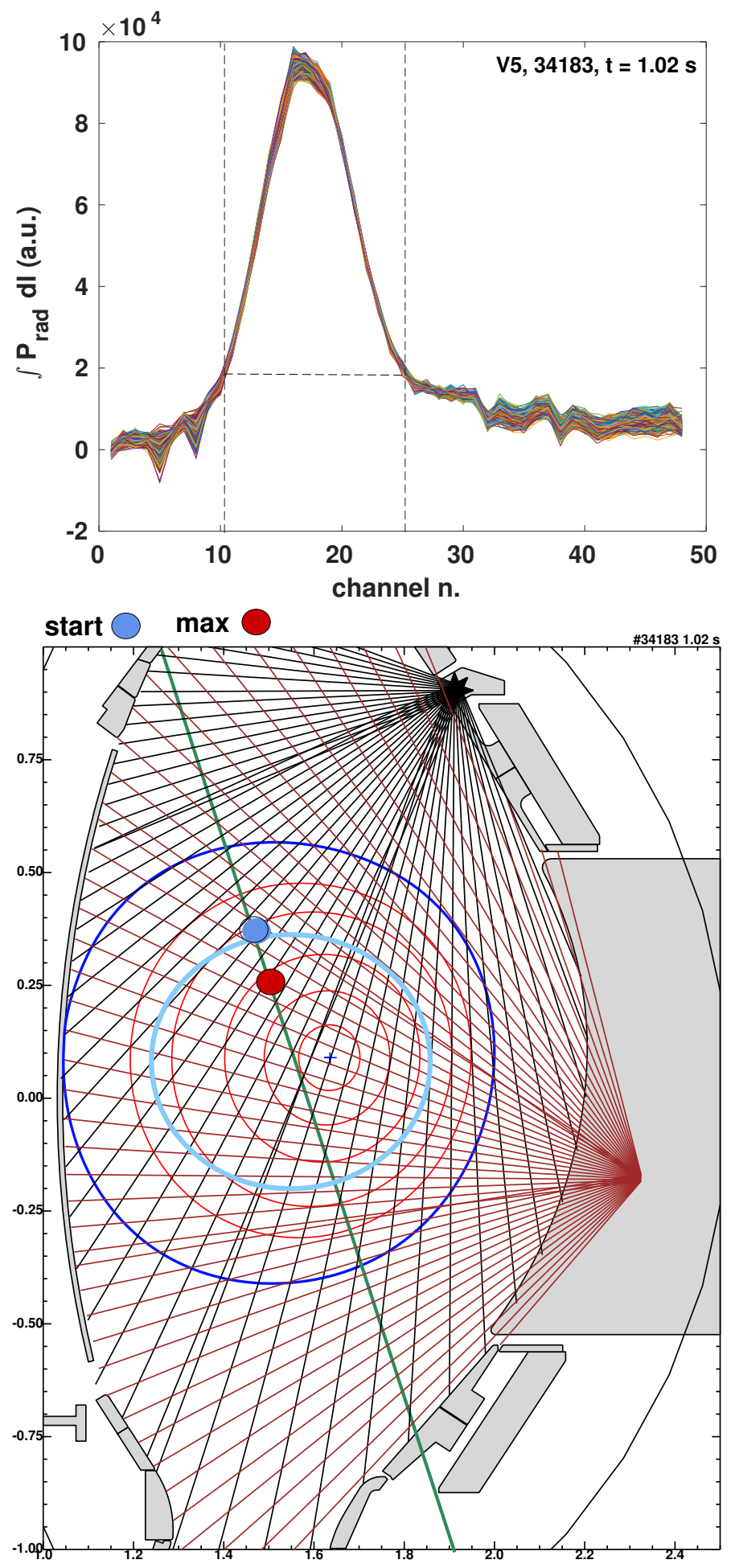

Figure 21: Top: Line integrated measurements from the vertical diode bolometer camera. The channels n. 10 and n. 25 are tangent to the blue circle drawn in the plasma centre (bottom figure). Bottom: Line of sight of the vertical and horizontal diode bolometer cameras in sector 5 . The blue and red points are the position of start and maximum of the radiation emission during pellet ablation, derived from figure 20. The oblique green line is the expected pellet trajectory (shot 34183). 

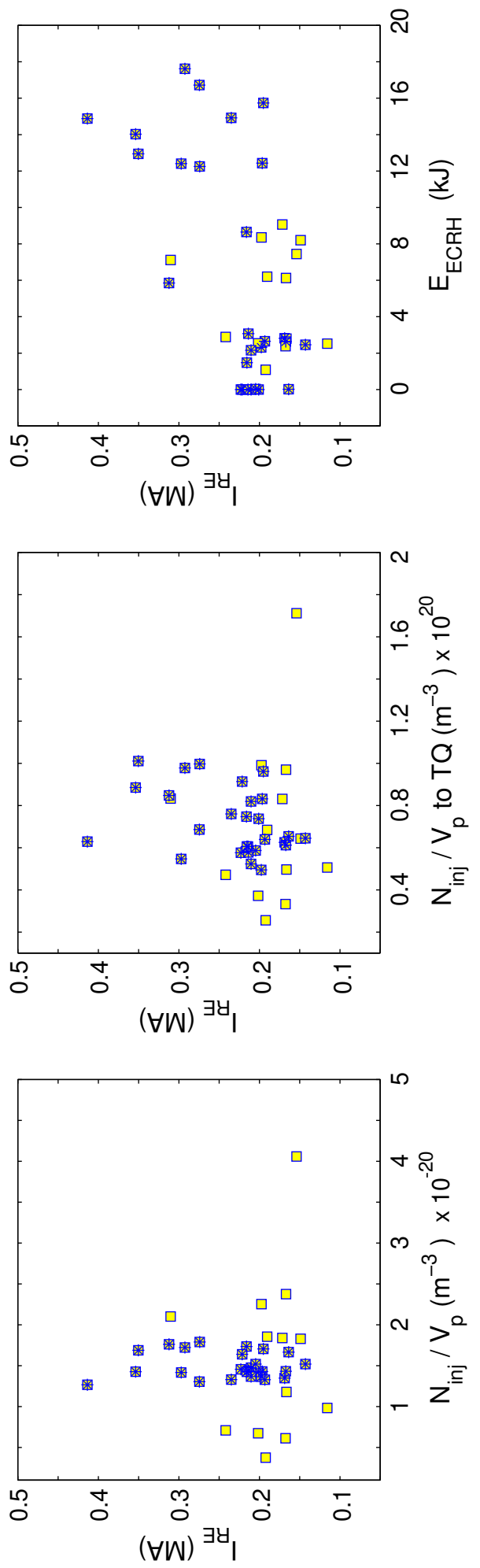

Figure 22: Initial RE current $\left(I_{R E}\right)$ versus (a) the number of injected gas atoms per plasma volume $\left(N_{i n j} / V_{p}\right)$; (b) the number of gas atoms per plasma volume injected up to the thermal quench; (c) the time integrated ECRH power injected after the MGI valve trigger generation time. The solid blue symbols pertain to discharges within the 1.2-1.8 $10^{20} \mathrm{~m}^{-3} N_{i n j} / V_{p}$ range. 

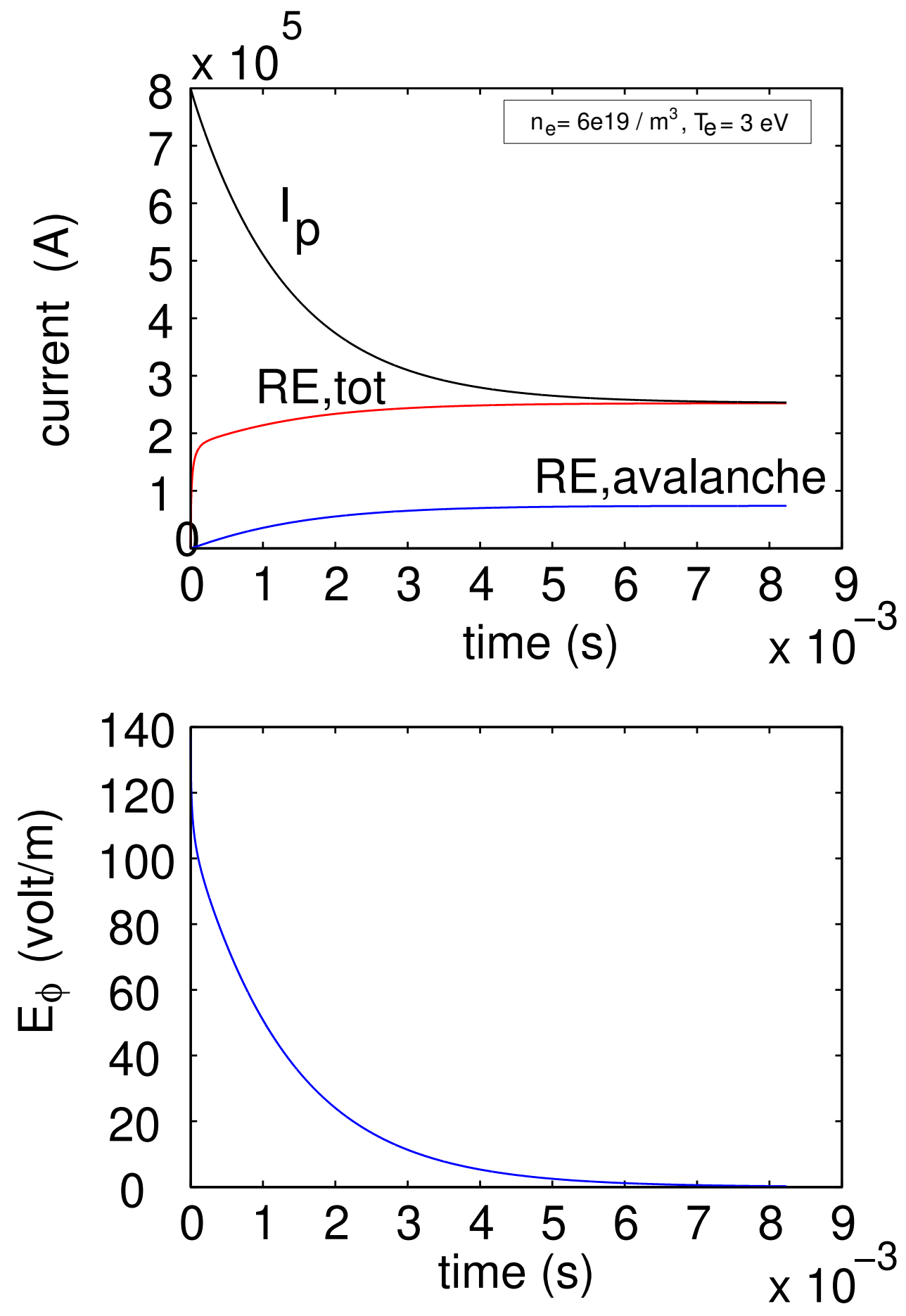

Figure 23: Time evolution (top) of the total plasma current $\left(I_{p}\right)$, of the total RE current (RE,tot), of the component of the RE current generated by avalanche and (bottom) of the toroidal electric field $\left(E_{\phi}\right)$ calculated by the 0D model. 


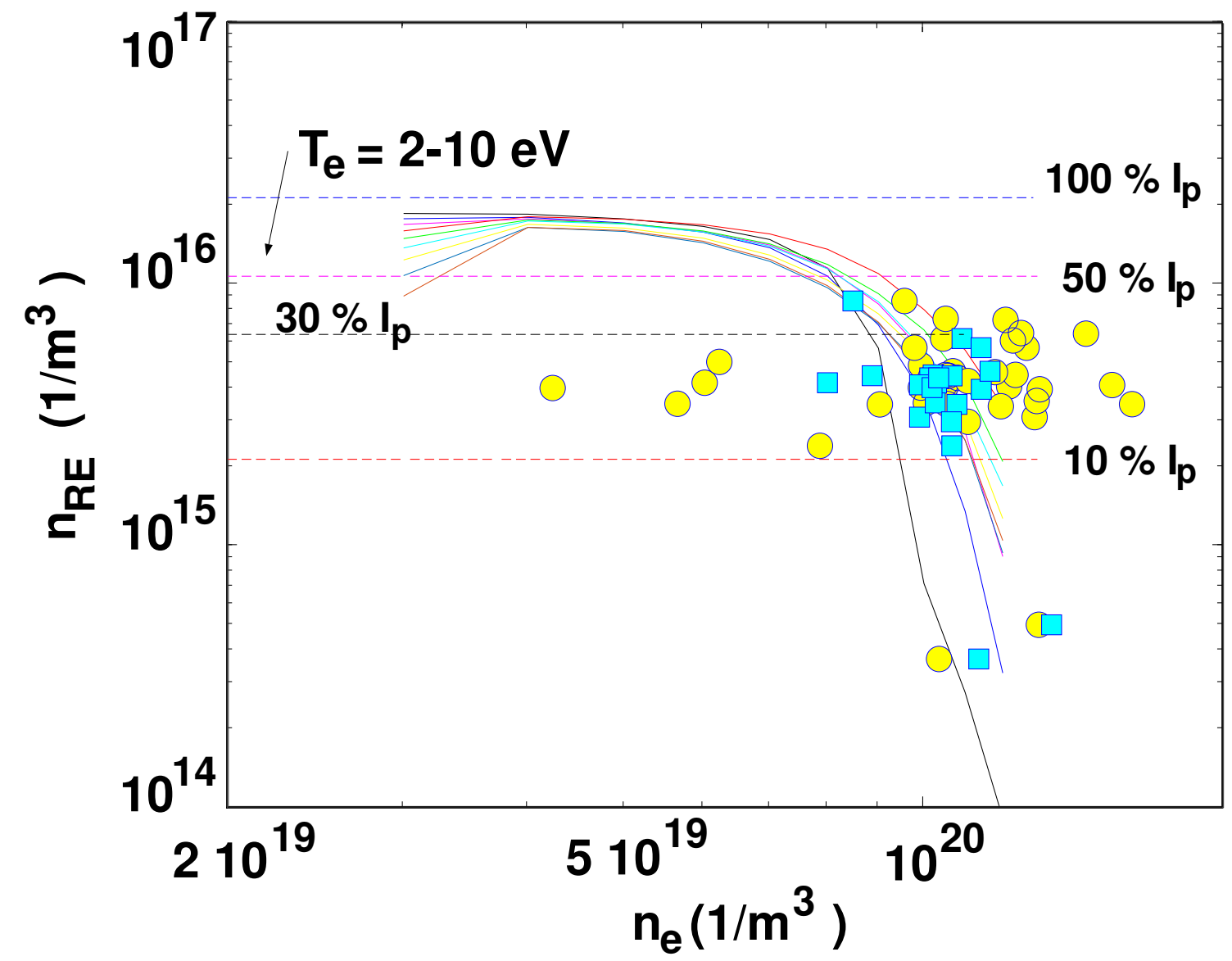

Figure 24: The different curves represent the RE density $\left(n_{R E}\right)$, calculated with the 0D model, versus the free electron density $\left(n_{e}\right)$. The electron temperature is varied in the range 2-10 eV. The symbols pertain to the inferred $n_{R E}$ versus the measured $n_{e}$. The square and circular symbols are used for discharges with correct $n_{e}$ measurements and calculated $n_{e}$ respectively. 

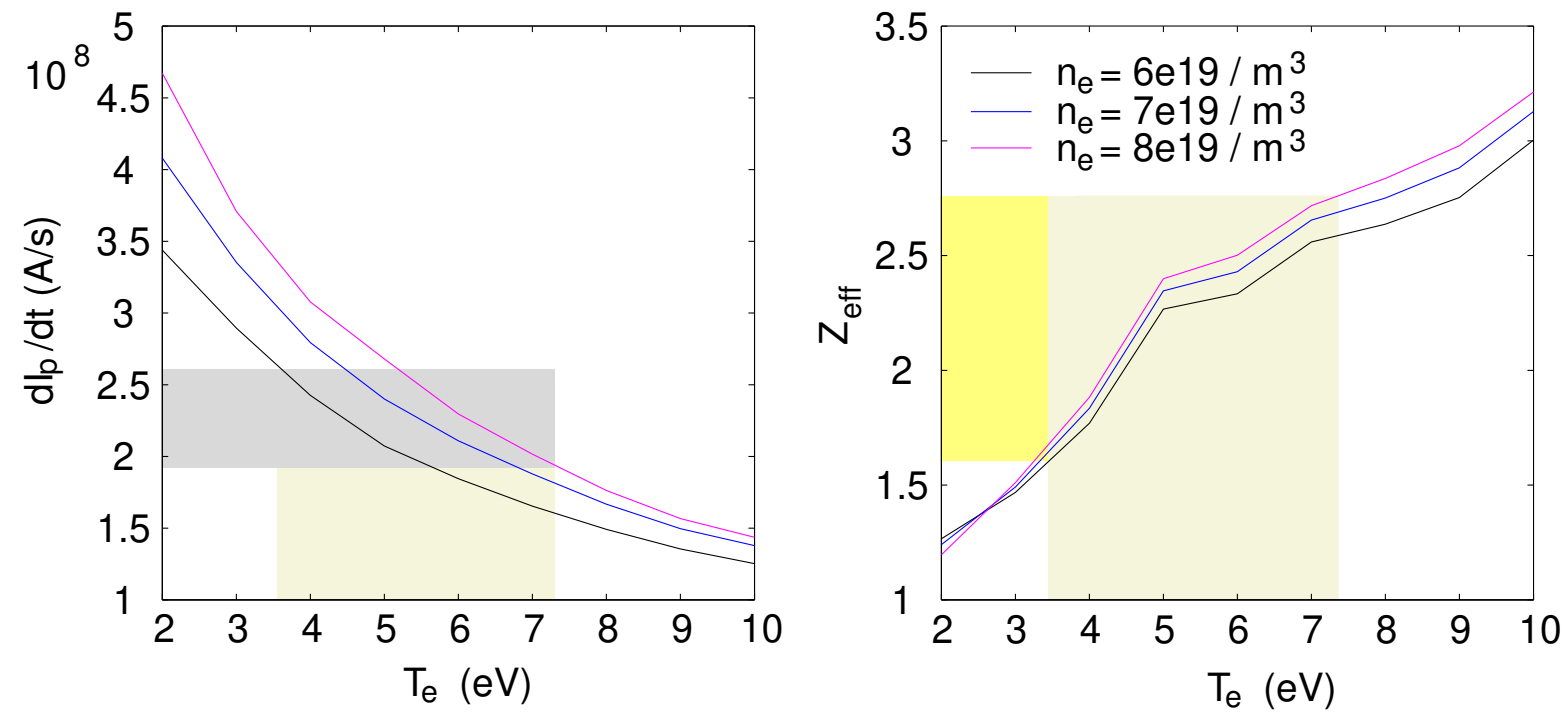

Figure 25: (Left) Current decay rate $\left(d I_{p} / d t\right)$ versus electron temperature $\left(T_{e}\right)$ and (right) the effective ion charge $\left(Z_{e f f}\right)$ versus $T_{e}$ for different values of the electron density $\left(n_{e}\right)$. 


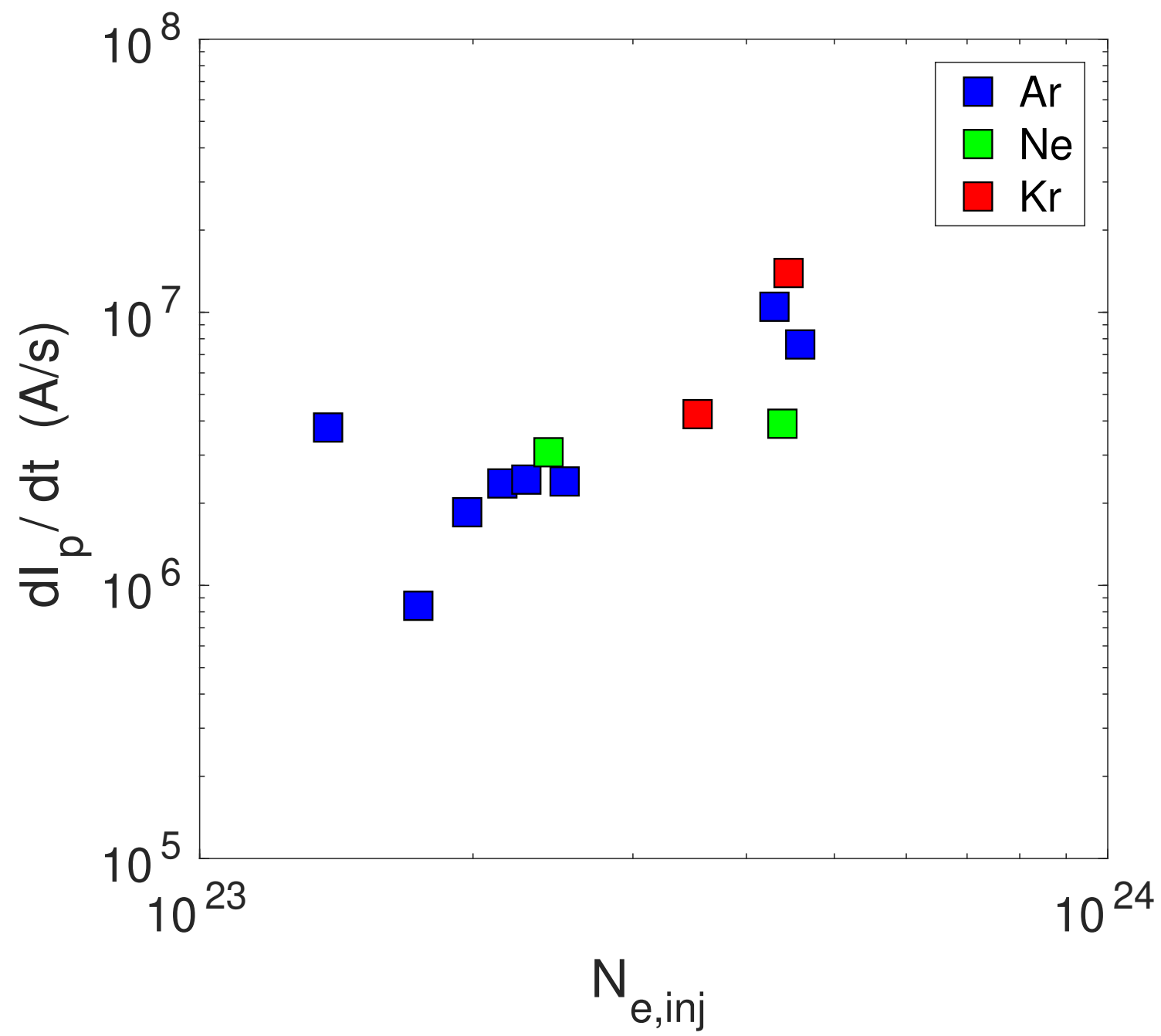

Figure 26: Speed of RE current decay, $d I_{p} / d t$, versus the total number of electrons (bound to the impurity atoms) injected, $N_{e, i n j}$. 

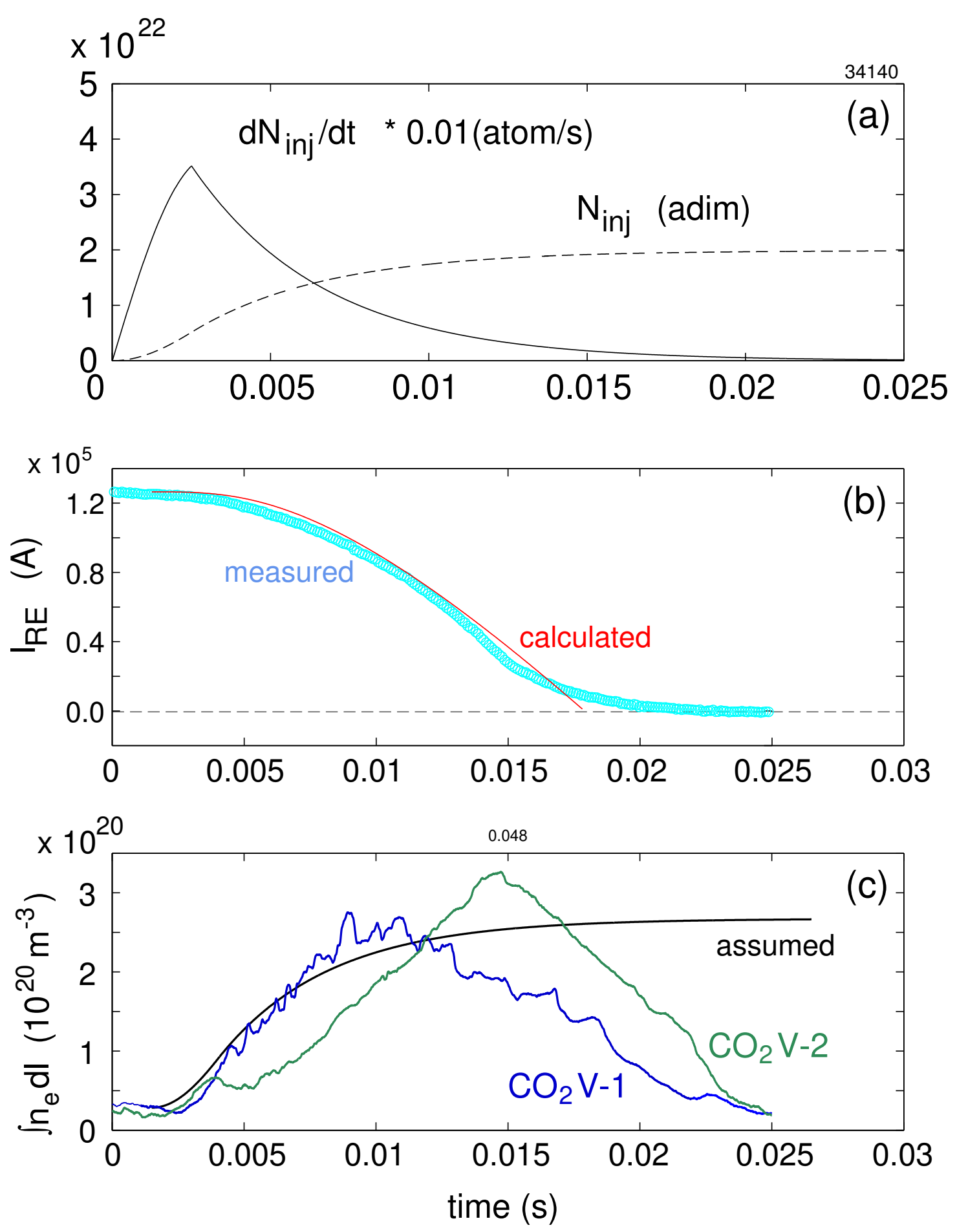

Figure 27: (a) The argon flux $\left(d N_{i n j} / d t\right)$ and the number of injected atoms $\left(N_{i n j}\right)$ as function of time. (b) Measured and calculated RE current $\left(I_{R E}\right)$. (c) Free electron density $\left(n_{e}\right)$ measured by the $\mathrm{CO}_{2}$ interferometer and assumed in the calculation (shot $34140)$. 

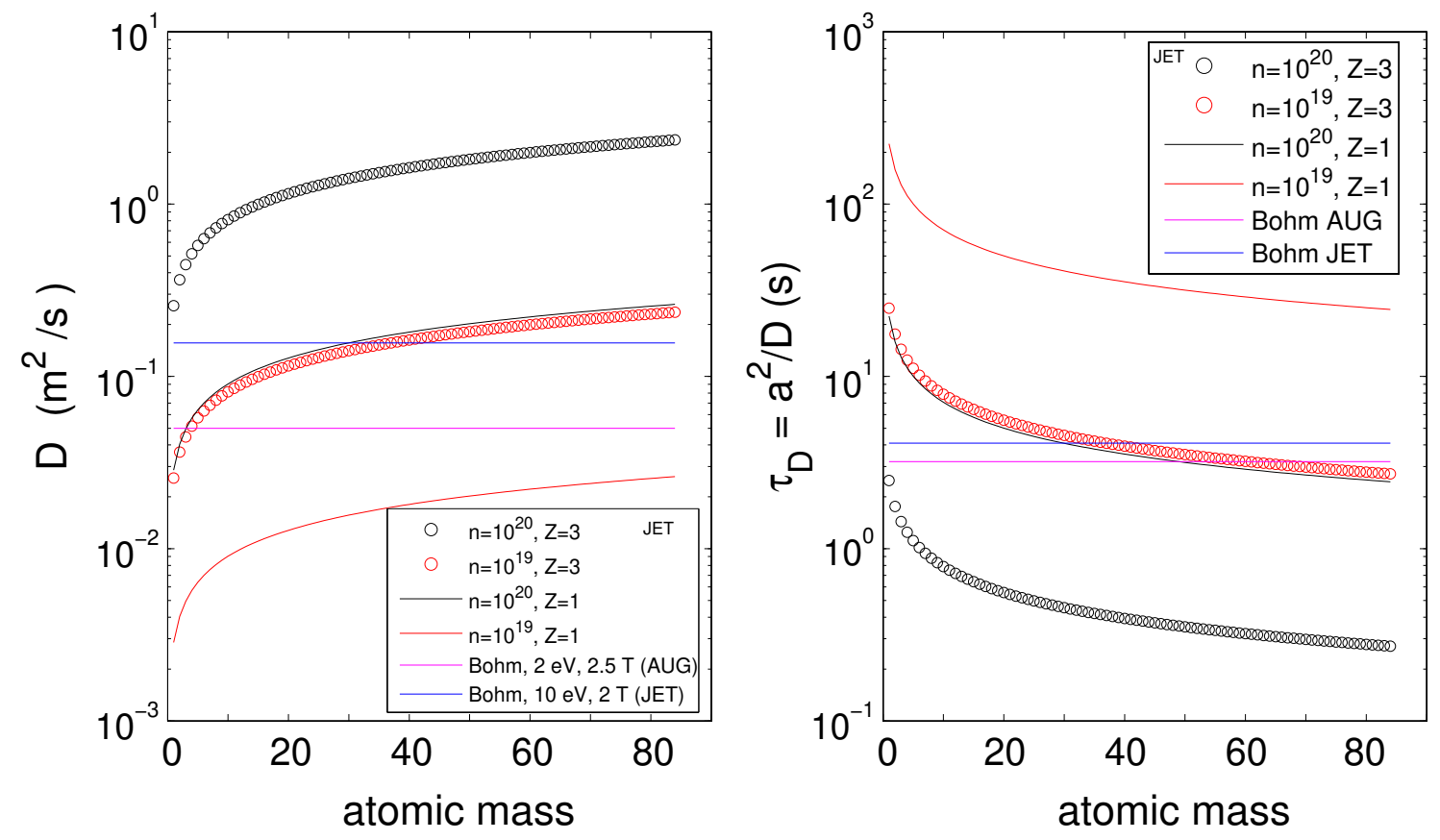

Figure 28: Dependence of the classical and of the Bohm's particle diffusion coefficient $(D)$ and of the relative diffusion times $\left(\tau_{D}\right)$ on the atomic mass. The different curves pertain to different values of the density $(n)$, of the ionization state $(Z)$, of the temperature $(T)$ and of the magnetic field (in Tesla, T) for JET. The Bohm's $D$ and $\tau_{D}$ are also evaluated for AUG. 